\title{
On the adoption of MC/DC and control-flow adequacy for a tight integration of program testing and statistical fault localization ${ }^{*, * *}$
}

\author{
Bo Jiang a , Ke Zhai ${ }^{\text {b }}$ W. K. Chan ${ }^{\mathrm{c}, \dagger}$, T. H. Tse ${ }^{\mathrm{b}}$, Zhenyu Zhang ${ }^{\mathrm{d}}$ \\ ${ }^{a}$ School of Computer Science and Engineering, Beihang University, Beijing, China \\ ${ }^{\mathrm{b}}$ Department of Computer Science, The University of Hong Kong, Pokfulam, Hong Kong \\ ${ }^{\mathrm{c}}$ Department of Computer Science, City University of Hong Kong, Tat Chee Avenue, Hong Kong \\ ${ }^{\mathrm{d}}$ State Key Laboratory of Computer Science, Institute of Software, Chinese Academy of Sciences, Beijing, China
}

\section{A B S T R A C T}

Context: Testing and debugging consume a significant portion of software development effort. Both processes are usually conducted independently despite their close relationship with each other. Test adequacy is vital for developers to assure that sufficient testing effort has been made, while finding all the faults in a program as soon as possible is equally important. A tight integration between testing and debugging activities is essential.

Objective: The paper aims at finding whether three factors, namely, the adequacy criterion to gauge a test suite, the size of a prioritized test suite, and the percentage of such a test suite used in fault localization, have significant impacts on integrating test case prioritization techniques with statistical fault localization techniques.

Method: We conduct a controlled experiment to investigate the effectiveness of applying adequate test suites to locate faults in a benchmark suite of seven Siemens programs and four real-life UNIX utility programs using three adequacy criteria, 16 test case prioritization techniques, and four statistical fault localization techniques. We measure the proportion of code needed to be examined in order to locate a fault as the effectiveness of statistical fault localization techniques. We also investigate the integration of test case prioritization and statistical fault localization with postmortem analysis.

Result: The main result shows that on average, it is more effective for a statistical fault localization technique to utilize the execution results of a MC/DC-adequate test suite than those of a branch-adequate test suite, and is in turn more effective to utilize the execution results of a branch-adequate test suite than those of a statement-adequate test suite. On the other hand, we find that none of the fault localization techniques studied can be sufficiently effective in suggesting fault-relevant statements that can fit easily into one debug window of a typical IDE.

Conclusion: We find that the adequacy criterion and the percentage of a prioritized test suite utilized are major factors affecting the effectiveness of statistical fault localization techniques. In our experiment, the adoption of a stronger adequacy criterion can lead to more effective integration of testing and debugging.

Keywords: Debugging, testing, adequacy criterion, MC/DC, branch coverage, fault localization, test case prioritization

\footnotetext{
* () 2013 Elsevier Inc. This material is presented to ensure timely dissemination of scholarly and technical work. Personal use of this material is permitted. Copyright and all rights therein are retained by authors or by other copyright holders. All persons copying this information are expected to adhere to the terms and constraints invoked by each author's copyright. In most cases, these works may not be reposted without the explicit permission of the copyright holder. Permission to reprint/republish this material for advertising or promotional purposes or for creating new collective works for resale or redistribution to servers or lists, or to reuse any copyrighted component of this work in other works must be obtained from Elsevier Inc.

** This research is supported in part by the General Research Fund of the Research Grants Council of Hong Kong (project nos. 111410 and 717811 ), a Strategic Research Grant of City University of Hong Kong (project no. 7002673), grants of the National Natural Science Foundation of China (project no. 61003027 and 61202077), and the Fundamental Research Fund for Central Universities (project no. YWF-12-LXGY-008). Part of this research was conducted when Tse was a visiting scholar at the State Key Laboratory of Computer Science, Institute of Software, Chinese Academy of Sciences, Beijing, China.

$\dagger$ Corresponding author.
} 


\section{Introduction}

Software testing can reveal program failures by running the program over a set of test cases. However, simply detecting program failures is inadequate. Developers must continue to debug the program, that is, to locate faults followed by fixing them. They also need to test the modified program to verify whether the identified fault has been removed as expected. Such an integrated loop of testing and debugging processes account for more than $30 \%$ of the total effort in typical software development [2]. Although testing and debugging techniques are under active research, a tight integration between testing and debugging is relatively understudied. A better understanding of such integration helps invent new methods that may further save project costs.

A fundamental problem in software testing is to know when to stop testing. Testers may apply a test adequacy criterion such as statement coverage to measure the progress of testing [1][5] and stop it accordingly. They may further use a test case prioritization technique [9][10][15][21][25] to reorder the test cases. As soon as the test adequacy criterion has been achieved, testers may stop applying further prioritized test cases even though the rest have been scheduled. Furthermore, if the executed test cases have exposed a program failure, testers may conduct debugging immediately. In such situations, there are a few dimensions that affect which particular test cases are used for subsequent debugging. They include the kind of test adequacy criterion used, the kind of test case prioritization technique used, and the proportion of prioritized test cases used.

There are many forms of debugging techniques. A major category under active research in the last decade is statistical fault localization [3][12][22][23][27][31][32]. They are usually based on the coverage statistics of a set of executions to assess which particular program entity is more faultrelevant than other entities in the same program. Previous studies [16][23] have found empirically that the effectiveness of statistical fault localization to identify faults is heavily affected by the size of the test suite and the strategies used to prioritize test cases. On the other hand, the aspect of test adequacy criterion has not been studied.

To study the issue of integration between testing and debugging, we investigate in this paper the above three dimensions of test suite composition (namely, test adequacy criterion, test case prioritization technique, and the proportion of prioritized test cases used) from the perspective of statistical fault localization. Because the code coverage information on the program under regression test achieved by different prioritization strategies (such as random, adaptive random [15], meta-heuristics [21], and greedy [9]) on different types of adequate test suites can be different, statistical fault localization techniques are likely to demonstrate different effectiveness when using the corresponding execution statistics to locate fault-relevant program entities. Jiang et al. [16][17] studied the effectiveness of using the prioritized test suites produced by different test case prioriti- zation strategies to locate faults by statistical fault localization techniques. In terms of the relative mean percentage of code examined to locate faults, they found empirically [17] that the random strategy and the additional statement strategies [9] can be more stable than the clustering-based and the total strategies. Nonetheless, they have not investigated the influence of the composition of adequate test suites that serve as inputs to test case prioritization and fault localization techniques. There are many important research questions remaining to be answered, such as the probability of obtaining a test suite that is both adequate with respect to some testing criterion and effective with respect to some fault localization technique, and whether the suggested suspicious region (that is, the list of statements suspected of containing a fault) can easily fit into a debug window on the canvas of a typical IDE, given that this suggestion is produced by a test suite that is deemed to be adequate.

In this paper, we report the results of an empirical study that applied three test adequacy criteria, 16 test case prioritization techniques, and four statistical fault localization techniques to 11 subject programs. We used Modified Condition/Decision Coverage (MC/DC) adequacy, branch adequacy, and statement adequacy because they are commonly practiced coverage criteria that can be applied to widely-used complex programs [1][5]. In total, we used 262 faulty program versions with 1000 different test suites for every adequacy criterion. Each prioritization technique was employed to prioritize each such test suite in every round of the best two test adequacy criteria (owing to our resource limitation in analyzing the huge amount of data). The corresponding execution statistics of the reordered test cases were utilized to locate faults using each of the four fault localization techniques. We also repeated the same procedure by systematically varying the proportion of each reordered test suite to simulate that only a portion of a reordered test suite can be used for fault localization. In total, we repeated such variations ten times for every test suite produced by each test case prioritization technique. As such, we have produced more than 330 million data points in terms of Expense [31]. It would be infeasible to report all the individual data in detail. Hence, we only report how well MC/DC adequacy, branch adequacy, and statement adequacy integrate with statistical fault localization and how they compare with one another at a summary level. We also studied whether the use of adequate test suites is more effective than random test suites in supporting fault localization.

Although many statistical fault localization research achievements have been obtained in the past decade, our empirical results still show many interesting findings. First, the use of MC/DC-adequate test suites is more effective in integrating with statistical fault localization than the use of branch-adequate test suites, which in turn is more effective than the use of statement-adequate test suites. Our result supports the conjecture that a stronger adequacy criterion supports statistical fault localization better. Second, the adoption of test case prioritization seems preferential, as supported by our result that shows no more than $30-40 \%$ of the test cases compromise the effectiveness of fault localiza- 
tion significantly in the statistical sense if the original test suites are adequate with respect to some test adequacy criterion. Last but not least, the result further shows that the fault localization techniques studied can still be ineffective in suggesting fault relevant statements that can easily fit into one debug window (e.g., 25 lines of code) in a typical IDE such as Eclipse. As such, we find that the current state of integration between testing and debugging techniques is still inadequate and far from satisfactory, which urges for more research.

This paper extends its conference version [14] in the following aspects:

(a) It reports the new result on $\mathrm{MC} / \mathrm{DC}$, a well-adopted adequacy criterion. It also reports the result of the use of the random strategy as a baseline "criterion" for comparison with the other three test adequacy criteria.

(b) It significantly extends the empirical study that includes a comprehensive study on how fault localization effectiveness in terms of the metric Expense varies against the change of size of test suites with respect to each test adequacy criterion.

(c) It proposes a metric SavingRate, which allows one to compare different adequacy criteria by normalizing an existing metric by the sizes of test suites.

(d) It analyzes our finding from the perspective of the subsumption relations among adequacy criteria in the integration of test case prioritization and statistical fault localization.

The main contribution of this paper with its preliminary studies [13][14] is as follows: (i) It presents the first controlled experiment to study the probability of obtaining a test suite that is both adequate with respect to a specific test adequacy criterion and effective with respect to a specific fault localization technique. (ii) It is the first study on the effectiveness of a hierarchy of adequacy criteria when their adequate test suites are utilized in statistical fault localization techniques. (iii) It proposes a new metric SavingRate that characterizes how effective an adequacy criterion is in the integration with statistical fault localization after discounting the impact of test suite sizes. (iv) It reports the first experimental results on how likely on average a test case prioritization technique may effectively integrate with a statement-level statistical fault localization technique if the original test suite is adequate with respect to a specific test adequacy criterion.

The rest of the paper is organized as follows: Section 2 reviews the test case prioritization and statistical fault localization techniques used in our study. We present our controlled experiment and its results in Section 3. Section 4 describes related work followed by a conclusion in Section 5.

\section{Background}

This section outlines the test case prioritization and statistical fault localization techniques applied in our empirical study.

\subsection{Test case prioritization techniques}

We follow [9] to categorize test case prioritization techniques in two dimensions. The first dimension is granularity, expressed in terms of program entities, which include statements, branches, and functions in the experiment. The second dimension is prioritization strategy. We study the Greedy [9] and the ART [15] strategies. The Greedy strategy can be further classified into the Total and Additional sub-strategies [9]. (We note that there are other types of greedy strategies, but they are not a part of our study, and hence we do not classify them here.) The $A R T$ strategy is reported in [15].

$A R T$ represents a strategy that randomly selects test cases followed by resolving the randomness among the selected test cases through a coverage measure. Greedy represents a strategy that selects test cases through a coverage measure followed by resolving tie cases randomly. As such, we refer to these two strategies as the coveragebefore-random $(C 2 R)$ strategy and the random-beforecoverage $(R 2 C)$ strategy, respectively. Table 1 summarizes the techniques studied in our experiment.

C2R strategy: When we pair up the two Greedy substrategies with the three levels of granularities, we produce six techniques: total statement (total-st), total branch (totalbr), total function (total-fn), additional statement (addtl-st), additional branch (addtl-br), and additional function (addtl$\mathrm{fn})$. All of them have been reported in previous work [9]. The total statement (total-st) test case prioritization technique ranks test cases in descending order of the number of statements that they cover. When two test cases cover the same number of statements, it orders them randomly. The total branch (total-br) and the total function (total-fn) test case prioritization techniques are the same as total-st, except that they use branch coverage and function coverage information, respectively, instead of statement coverage information. The additional statement (addtl-st) test case prioritization technique is the same as total-st, except that in each round, it selects a test case that covers the maximum number of statements not yet exercised. When no remaining test case in the test suite can further improve the statement coverage, addtl-st resets all the statements to "not yet covered" and reapplies the same procedure to the set of remaining test cases. When two test cases cover the same number of additional statements in a round, it randomly picks one. The additional branch (addtl-br) and additional function (addtl-fn) test case prioritization techniques are the same as addtl-st, except that they use branch coverage and function coverage data, respectively, rather than statement coverage data.

R2C Strategy: As mentioned above, we use the $A R T$ strategy [15] to represent the R2C strategy. The basic algorithm of $A R T$ reorders the test cases by iteratively constructing a candidate set of test cases, and then picks one test case out of the candidate set until all the test cases in a given regression test suite have been selected. To generate a candidate set of test cases, the algorithm randomly adds the not-yet-selected test cases one by one into the candidate set (which is initially empty) as long as they can increase the 
code coverage achieved by the candidate set. The algorithm then selects a test case from the candidate set that maximizes the distance of the test cases from the already selected test cases. The distance between two test cases is defined as the Jaccard distance between the coverage of the program entities of the two test cases. By combining three distance measures (average, minimum, and maximum) and the above three levels of granularities, we produce nine techniques: ART-st-maxmin, ART-st-maxavg, ART-st-maxmax, ART-fn-maxmin, ART-fn-maxavg, ART-fn-maxmax, ART-br-maxmin, ART-br-maxavg, and ART-brmaxmax. All the nine techniques have been defined and evaluated in our previous work [15].

Table 1 Test case prioritization techniques.

\begin{tabular}{|c|c|c|c|}
\hline \multicolumn{2}{|c|}{ Ref. } & Acronym & Brief Description \\
\hline \multicolumn{2}{|c|}{$T 1$} & Random & Random ordering \\
\hline \multicolumn{2}{|c|}{ Ref. } & Acronym & Brief Description \\
\hline \multirow{6}{*}{ 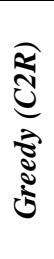 } & $T 2$ & total-st & Total statement \\
\hline & $T 3$ & total-fn & Total function \\
\hline & $T 4$ & total-br & Total branch \\
\hline & $T 5$ & addtl-st & Additional statement \\
\hline & $T 6$ & addtl-fn & Additional function \\
\hline & $T 7$ & addtl-br & Additional branch \\
\hline
\end{tabular}

\begin{tabular}{|c|c|c|c|c|}
\hline \multicolumn{2}{|c|}{ Ref. } & Acronym & Level of Coverage & Test Set Distance \\
\hline \multirow{9}{*}{ 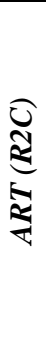 } & $T 8$ & ART-st-maxmin & \multirow{3}{*}{ Statement } & Maximize the minimum distance between test cases \\
\hline & $T 9$ & ART-st-maxavg & & Maximize the average distance between test cases \\
\hline & $T 10$ & ART-st-maxmax & & Maximize the maximum distance between test cases \\
\hline & $T 11$ & ART-fn-maxmin & \multirow{3}{*}{ Function } & Maximize the minimum distance between test cases \\
\hline & $T 12$ & ART-fn-maxavg & & Maximize the average distance between test cases \\
\hline & $T 13$ & ART-fn-maxmax & & Maximize the maximum distance between test cases \\
\hline & $T 14$ & ART-br-maxmin & \multirow{3}{*}{ Branch } & Maximize the minimum distance between test cases \\
\hline & $T 15$ & ART-br-maxavg & & Maximize the average distance between test cases \\
\hline & $T 16$ & ART-br-maxmax & & Maximize the maximum distance between test cases \\
\hline
\end{tabular}

Table 2 Statistical fault localization techniques.

\begin{tabular}{|l|c|}
\hline \multicolumn{1}{|c|}{ Technique } & Ranking formula \\
\hline Tarantula [19] & $\frac{\% \text { failed }(s)}{\% \text { failed }(s)+\% \text { passed }(s)}$ \\
\hline Adapted Statistical Bug Isolation (SBI) [22] & $\frac{\text { failed }(s)}{\text { Tie-breaker: max }(\% \text { failed }(s) \text { \%passed }(s))}$ \\
\hline Jaccard [3] & $\frac{\text { failed }(s)}{\text { totalfailed }+ \text { passed }(s)}$ \\
\hline Ochiai [3] & $\frac{\text { failed }(s)}{\sqrt{\text { totalfailed } \times(\text { failed }(s)+\text { passed }(s))}}$ \\
\hline
\end{tabular}

\subsection{Fault localization techniques}

We revisit four statistical fault localization techniques used in our study. Each of them computes the suspiciousness of individual statements, followed by ranking these statements according to their suspiciousness scores. One of the techniques, namely Tarantula [19], further uses a tiebreaker to resolve statements having identical suspiciousness values so that the ranking can be fine-tuned. This set of techniques was also used in the experiment in previous work [29].

Table 2 summarizes these fault localization techniques. The function \%failed $(s)$ in the table is the percentage of failed test cases that execute a statement $s$ (among all the failed test cases in the test suite). The function \%passed(s) is the percentage of passed test cases that execute that statement. The functions failed $(s)$ and passed $(s)$ calculate 
the number of failed and passed test cases, respectively, that exercises statement $s$. The variable totalfailed is the total number of failed test cases.

\section{Controlled Experiment}

In this section, we report on our controlled experiment.

\subsection{Research questions}

We raised three new and important research questions to study the issue of integrating testing and debugging in relation to test adequacy.

$\boldsymbol{R Q 1 :}$ How likely is it that a test suite produced to satisfy a test adequacy criterion is effective for fault localization?

RQ2: How do different test adequacy criteria compare with one another after factoring out the impact of the test suite size?

RQ3: After prioritizing the test cases in an effective test suite, what minimum portion of this test suite can have the same fault localization effectiveness as the whole adequate suite?

From the study of RQ1, developers and researchers will have a better understanding of the probability of generating effective test suites based on test adequacy criteria with respect to some of the best and representative statistical fault localization techniques. Having a high probability makes developers more comfortable in employing such adequate test suites to perform regression testing on their programs and using the execution statistics to aid fault localization activities. On the other hand, if the probability is found to be low, the result can alert developers when using such test suites. They may consider improving the test suites before use to support their testing and debugging activities. For RQ1, we used test suites of various sizes that satisfy a specific testing adequacy criterion. This is because we want to evaluate the "absolute" fault localization effectiveness of the test suites generated by different adequacy criteria. Thus, evaluating RQ1 without taking test suite size into consideration helps us better understand the fault localization capability of adequate test suites in practice.

Answering RQ2 will enable us to gauge the relative fault localization effectiveness of the test suites generated by different test adequacy criteria. Different adequacy criteria will inherently require different test suite sizes, which is a known factor affecting fault localization effectiveness. Adding more test cases to a smaller adequate test suite will introduce redundancy with respect to the test adequacy criterion used to produce the test suite, which makes the assessment of test adequacy criteria not meaningful. Similarly, removing some test cases from a larger adequate test suite will make the latter inadequate with respect to the test adequacy criterion. Again, by so doing, it makes the assessment of test adequacy criteria not meaningful. As such, in the data analysis, we normalize the test suite sizes to make the comparison among different test adequacy criteria fairer.

Answering RQ3 helps developers and researchers decide whether the effort on prioritizing test cases is worthwhile and whether executing only the higher priority portion of the prioritized test cases still retains good fault localization effectiveness. If the finding is positive, developers may be comfortable in using a portion of test data for fault localization. On the other hand, if the finding is negative, additional test cases must be used to prevent the fault localization effectiveness of the test suites from being seriously compromised.

\subsection{Subject programs}

Our experiments used the Siemens suite and four UNIX programs as subjects, as shown in Table 3. For each subject program, the table shows the name, the number of faulty versions, the executable lines of code, the test pool size, and the average percentage of compound Boolean expressions in decisions in relation to all Boolean expressions in decisions. It is the existence of compound Boolean expressions in the decision statements in a program that makes the MC/DC adequacy criterion different from the branch adequacy criterion. All the subject programs were downloaded from the Software-artifact Infrastructure Repository (SIR) [7]. Each subject came with, among other files, a set of faulty versions and a test pool. In this work, we use single fault versions of the subject programs to study the proposed research questions.

\subsection{Test adequacy criteria}

We used three adequacy criteria, namely, statement adequacy, branch adequacy, and MC/DC adequacy. We chose branch coverage and statement coverage because they are commonly used criteria that are widely applicable to industrial-strength programs [5]. Moreover, many existing industrial-strength tools (such as gcov) can provide profiling data for testers to determine whether these two coverage criteria have been achieved. We also chose the MC/DC adequacy criterion because it is a well-adopted adequacy criterion in the aeronautics industry. For instance, MC/DC is used in the FAA's DO-178 standard [1] to ensure that the most safety-critical software is tested adequately. Thus, a study of the three adequacy criteria can provide valuable contribution to the advance in the state of the art in research and the advance in the state of the practice in industry.

The statement (or branch, respectively) adequacy criterion ensures that each statement (or branch, respectively) is covered at least once by a test suite. The Modified Condition/Decision Coverage (MC/DC) adequacy criterion [1] requires a number of conditions: (i) for each decision statement $d$, every point of entry or exit should be tested at least once, (ii) every condition in $d$ has taken all possible outcomes at least once, (iii) every decision statement in the program has taken all possible outcomes at least once, and (iv) each condition in $d$ has been shown to independently affect the outcome of $d$. A condition is shown to independently affect the outcome of a decision by varying only that condition while all the other possible conditions remain unchanged. To make the study complete, we also included the random strategy as a baseline criterion for comparison. 
Table 3 Subject programs.

\begin{tabular}{|c|c|c|c|c|c|}
\hline Group & Subject & No. of Faulty Versions & LOC & Test Pool Size & Percentage of Compound Boolean \\
\hline \multirow{7}{*}{ 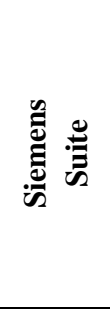 } & tcas & 41 & $133-137$ & 1608 & 2.4 \\
\hline & schedule & 9 & $291-294$ & 2650 & 3.2 \\
\hline & schedule2 & 10 & $261-263$ & 2710 & 1.0 \\
\hline & tot_info & 23 & $272-274$ & 1052 & 5.6 \\
\hline & print_tokens & 7 & $341-342$ & 4130 & 1.7 \\
\hline & print_tokens2 & 10 & $350-354$ & 4115 & 5.4 \\
\hline & replace & 32 & $508-515$ & 5542 & 2.0 \\
\hline \multirow{4}{*}{ 宏 } & flex (2.4.7-2.5.4) & 21 & $8571-10124$ & 567 & 5.5 \\
\hline & grep (2.2-2.4.2) & 17 & $8053-9089$ & 809 & 14.1 \\
\hline & gzip (1.1.2-1.3) & 55 & $4081-5159$ & 217 & 11.6 \\
\hline & sed (1.18-3.02) & 17 & 4756-9289 & 370 & 11.6 \\
\hline
\end{tabular}

Table 4 Levels of adequacies achieved for the subject programs.

\begin{tabular}{|l|c|c|c|c|c|c|}
\hline \multirow{2}{*}{ Subject } & \multicolumn{2}{|c|}{ Statement } & \multicolumn{2}{c|}{ Branch } & \multicolumn{2}{c|}{ MC/DC } \\
\cline { 2 - 7 } & Mean & Standard Deviation & Mean & Standard Deviation & Mean & Standard Deviation \\
\hline Tcas & $95 \%$ & $5 \%$ & $96 \%$ & $5 \%$ & $95 \%$ & $3 \%$ \\
\hline schedule & $96 \%$ & $4 \%$ & $97 \%$ & $5 \%$ & $94 \%$ & $6 \%$ \\
\hline schedule2 & $96 \%$ & $6 \%$ & $95 \%$ & $8 \%$ & $95 \%$ & $7 \%$ \\
\hline tot_info & $98 \%$ & $6 \%$ & $98 \%$ & $3 \%$ & $97 \%$ & $4 \%$ \\
\hline print_tokens & $99 \%$ & $5 \%$ & $99 \%$ & $2 \%$ & $95 \%$ & $6 \%$ \\
\hline print_tokens2 & $99 \%$ & $3 \%$ & $97 \%$ & $4 \%$ & $95 \%$ & $5 \%$ \\
\hline replace & $98 \%$ & $5 \%$ & $98 \%$ & $3 \%$ & $94 \%$ & $6 \%$ \\
\hline flex 2.4.7-2.5.4 & $97 \%$ & $6 \%$ & $96 \%$ & $8 \%$ & $98 \%$ & $4 \%$ \\
\hline grep 2.2-2.4.2 & $95 \%$ & $3 \%$ & $97 \%$ & $6 \%$ & $93 \%$ & $3 \%$ \\
\hline gzip 1.1.2-1.3 & $98 \%$ & $3 \%$ & $98 \%$ & $4 \%$ & $96 \%$ & $4 \%$ \\
\hline
\end{tabular}

For the Siemens programs, SIR provides a set of 1000 branch-adequate test suites, 1000 statement-adequate test suites, and 1000 random test suites. We further constructed $1000 \mathrm{MC} / \mathrm{DC}$-adequate test suites using the test pool for each Siemens program. However, only one test pool is available for each UNIX program. We used this test pool to construct 1000 branch-adequate test suites, 1000 statementadequate test suites, and $1000 \mathrm{MC} / \mathrm{DC}$-adequate test suites for each UNIX program. The test suites are constructed independently of one another, while a test case may belong to more than one test suite. The sizes of the test suites for all programs range from 16 to 289 . To generate a MC/DCadequate test suite, we iteratively selected test cases from the test pool and added them into the test suite if the coverage achieved by the constructed test suite could be improved in terms of the criterion. The means and standard deviations of the levels of adequacies achieved by different adequacy suites generated in our experiment are shown in Table 4. For example, the mean percentage of statement coverage achieved by all the generated suites for tcas is $95 \%$ with a standard deviation 5\%. When generating random test suites for the UNIX programs, we simply selected test cases randomly from the test pool until the required suite size has been reached. We randomly picked a value as the size of each random test suite to be constructed, and produced 1000 random test suites.

\subsection{Metrics}

\subsubsection{Expense}

To measure the effectiveness of fault localization, we used the Expense metric [12] defined as:

$$
\text { Expense }=\frac{\text { rank of the faulty statement }}{\text { total number of executable statements }}
$$

where the rank of a given statement is the sum of (a) the number of statements with higher suspiciousness values and (b) the number of statements with identical suspiciousness values and identical or higher tiebreaker values.

\subsubsection{FLSP}

In practice, a developer may only walk through a small portion of the source code. As a result, a high value of Expense (such as 90\%) may be useless for debugging. A sequence of test cases with respect to a fault localization technique and a given faulty program is said to be $\alpha$ effective if the value of Expense using this sequence of test cases by the fault localization technique on the faulty program is strictly lower than the threshold value specified by $\alpha$.

If this is the case, we say that "the test suite supports the fault localization technique". As such, the proportion of adequate test suites for a test adequacy criterion $C$ empirically represents the probability that a $C$-adequate test suite 
supports a statistical fault localization technique $T$. We use this probability to measure how well $C$ supports $T$.

Given a faulty program and a fault localization technique $T$, we define the metric Fault Localization Success Percentage (FLSP) as the ratio between the number of $\alpha$ effective test suites in a test suite pool $P$ and the size of $P$ (denoted by $|\mathrm{P}|$ ), thus:

$$
F L S P(T, P, \alpha)=\frac{\mid\{t \in P \mid t \text { is } \alpha \text {-effective }\} \mid}{|P|}
$$

\subsubsection{SavingRate}

We define Saving as the percentage of code that need not be examined to locate a fault.

$$
\text { Saving }=1-\text { Expense }
$$

Thus, the higher the value of Saving, the better will be the fault localization result.

However, when discussing the impact of an adequate test suite on statistical fault localization, an important factor to be considered is the number of test cases in the suite. Generally speaking, if a test adequacy criterion $A$ subsumes another criterion $B$, satisfying criterion $A$ will require more (if not the same) test cases than satisfying criterion $B$. The number of test cases within a test suite is, however, a confounding factor in comparing fault localization effectiveness achieved by different test suites. To compare the effectiveness of different test adequacy criteria in a more objective manner, it is important to control the impact of the test suite size.

We propose a derived metric SavingRate to measure the average effectiveness of a testing criterion in supporting statistical fault localization. Given a test suite of size $n$, we define SavingRate by the formula:

$$
\text { SavingRate }=\frac{\text { Saving }}{n}
$$

Intuitively, SavingRate is a measure of the fault localization effectiveness per test case. It is a measure of the fault localization capability of different adequacy criteria normalized against test suite size.

\subsection{Experimental setup}

We applied each test case prioritization technique (see Table 1) and each fault localization technique (see Table 2) to every adequate test suite of every subject program. For every prioritized test suite generated by each test case prioritization technique, we repeated the above procedure using, in turn, the top $10 \%, 20 \%, \ldots, 90 \%$ of the prioritized test suite. For every such portion of all the prioritized test suites applicable to every corresponding version of each subject program, we collected the values of Expense for each fault localization technique, and computed the FLSP values and the SavingRate values.

We conducted the experiment on a Dell PowerEdge 2950 server serving a Solaris UNIX system. We used gcc version 4.4.1 as the $\mathrm{C}$ compiler. The server has two Xeon 5430 (2.66 GHz, 4 core) processors and 4 GB physical memory. We followed previous test case prioritization studies [31] to remove faulty versions that cannot be detected by any test case in the test pool as well as those that can be detected by more than $20 \%$ of the test cases in the pool. We used the gcov tool with the gcc compiler to collect the execution statistics for each execution.

To study RQ1 and RQ2, we used all the random, branchadequate, statement-adequate, and MC/DC-adequate test suites for our experimentation. For each faulty version, we also removed those test suites that cannot detect the fault because the fault localization techniques we used require at least one failed test case. We also removed all the test suites whose results on our platform differed from those indicated in the downloaded benchmark. We then passed the execution statistics to all the four fault localization techniques. For RQ1, we followed [13] to measure their results in terms of FLSP on all subject programs with three different fault localization effectiveness threshold values $(1 \%, 5 \%$, and $10 \%)$. For RQ2, we calculated the SavingRate for all test suites generated from all adequacy criteria and all subject programs.

We studied RQ1 and RQ2 separately rather than merging them because they are targeting at different goals. For RQ1, we used the test suites satisfying a specific testing adequacy criterion without considering test suite size. In this way, we can evaluate the "absolute" fault localization effectiveness of the test suites generated by different adequacy criteria in practice. For RQ2, we are interested in comparing different adequacy criteria while controlling the confounding factor of test suite size. This makes the comparison between different adequacy criteria fairer.

RQ3 is a follow-up research question based on the results of RQ1 and RQ2. We used the two best adequacy criteria from the two previous research questions, namely, branch adequacy and MC/DC adequacy. In this way, we can still preserve the generality of our findings while controlling the scale of our empirical study. Similarly to RQ1 and RQ2, we removed all the test suites that contain no failed test case as well as all test suites that cannot work on our platform.

All ART techniques are adapted from random selection. We followed [13] to repeat each of them 20 times so as to obtain an average performance and to select 50 suites out of the available 1000 test suites for every Siemens and UNIX subject program. Thus, we conducted a total of 1000 prioritizations for every ART technique on each subject. We then used MATLAB for ANOVA tests and multiple comparisons on the mean values, and specified a 5\% significance level for hypothesis testing.

\subsection{Data analysis}

\subsubsection{Answering RQ1}

We studied the effectiveness of a fault localization technique using all the test cases within an adequate or random test suite. As a result, we need not distinguish between different test case prioritization techniques, as the test suites generated by them will have the same fault localization results. Tables 5 to 12 present the means and standard deviations of the numbers of effective suites averaged over all faulty versions of the Siemens and UNIX programs on 
Table 5 Mean numbers of effective test suites for Tarantula.

\begin{tabular}{|c|c|c|c|c|c|c|c|c|c|c|c|c|}
\hline Threshold Value & \multicolumn{4}{|c|}{$\alpha=1 \%$} & \multicolumn{4}{|c|}{$\alpha=5 \%$} & \multicolumn{4}{|c|}{$\alpha=10 \%$} \\
\hline Adequacy Criteria & Rand & Stmt & $\mathrm{Br}$ & $\mathrm{MC} / \mathrm{DC}$ & Rand & Stmt & $\mathrm{Br}$ & $\mathrm{MC} / \mathrm{DC}$ & Rand & Stmt & $\mathrm{Br}$ & $\mathrm{MC} / \mathrm{DC}$ \\
\hline tcas & 5 & 8 & 25 & 34 & 23 & 36 & 191 & 221 & 23 & 31 & 193 & 212 \\
\hline replace & 32 & 46 & 145 & 166 & 77 & 126 & 342 & 363 & 107 & 140 & 381 & 399 \\
\hline tot_info & 59 & 74 & 145 & 160 & 82 & 140 & 331 & 349 & 102 & 181 & 430 & 470 \\
\hline schedule & 0 & 1 & 14 & 18 & 37 & 52 & 169 & 198 & 45 & 61 & 243 & 281 \\
\hline schedule2 & 0 & 0 & 0 & 0 & 4 & 5 & 35 & 45 & 9 & 11 & 107 & 123 \\
\hline print_tokens & 1 & 3 & 55 & 59 & 17 & 22 & 151 & 178 & 21 & 37 & 215 & 247 \\
\hline print_tokens 2 & 18 & 56 & 156 & 163 & 83 & 121 & 317 & 361 & 101 & 159 & 332 & 387 \\
\hline grep & 121 & 371 & 618 & 719 & 348 & 546 & 832 & 867 & 432 & 756 & 936 & 962 \\
\hline sed & 108 & 319 & 604 & 732 & 401 & 594 & 860 & 886 & 511 & 753 & 931 & 971 \\
\hline flex & 124 & 376 & 665 & 750 & 335 & 586 & 827 & 890 & 486 & 720 & 951 & 979 \\
\hline gzip & 131 & 395 & 674 & 681 & 362 & 516 & 843 & 832 & 502 & 711 & 945 & 978 \\
\hline
\end{tabular}

Table 6 Standard deviations of the numbers of effective test suites for Tarantula.

\begin{tabular}{|c|c|c|c|c|c|c|c|c|c|c|c|c|}
\hline \multirow{2}{*}{$\begin{array}{l}\text { Threshold Value } \\
\text { Adequacy Criteria }\end{array}$} & \multicolumn{4}{|c|}{$\alpha=1 \%$} & \multicolumn{4}{|c|}{$\alpha=5 \%$} & \multicolumn{4}{|c|}{$\alpha=10 \%$} \\
\hline & Rand & Stmt & $\mathrm{Br}$ & $\mathrm{MC} / \mathrm{DC}$ & Rand & Stmt & $\mathrm{Br}$ & $\mathrm{MC} / \mathrm{DC}$ & Rand & Stmt & $\mathrm{Br}$ & $\mathrm{MC} / \mathrm{DC}$ \\
\hline tcas & 2 & 4 & 3 & 5 & 6 & 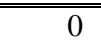 & 4 & 5 & 4 & 3 & 6 & 4 \\
\hline replace & 6 & 7 & 6 & 7 & 5 & 5 & 6 & 7 & 10 & 8 & 6 & 4 \\
\hline tot_info & 4 & 5 & 8 & 9 & 13 & 3 & 7 & 9 & 5 & 7 & 8 & 6 \\
\hline schedule & 0 & 0 & 3 & 2 & 3 & 5 & 4 & 5 & 4 & 4 & 3 & 3 \\
\hline schedule2 & 0 & 0 & 0 & 0 & 0 & 0 & 2 & 3 & 2 & 2 & 3 & 4 \\
\hline print_tokens & 0 & 1 & 4 & 6 & 3 & 3 & 1 & 4 & 3 & 4 & 6 & 4 \\
\hline print_tokens 2 & 5 & 6 & 5 & 3 & 7 & 8 & 7 & 6 & 5 & 5 & 2 & 5 \\
\hline grep & 30 & 26 & 28 & 20 & 25 & 21 & 26 & 28 & 27 & 24 & 22 & 32 \\
\hline sed & 22 & 27 & 22 & 23 & 26 & 29 & 23 & 29 & 30 & 27 & 23 & 33 \\
\hline flex & 25 & 31 & 19 & 18 & 28 & 33 & 32 & 34 & 16 & 18 & 12 & 18 \\
\hline gzip & 26 & 33 & 31 & 31 & 28 & 30 & 34 & 26 & 21 & 19 & 10 & 12 \\
\hline
\end{tabular}

Table 7 Mean numbers of effective test suites for SBI.

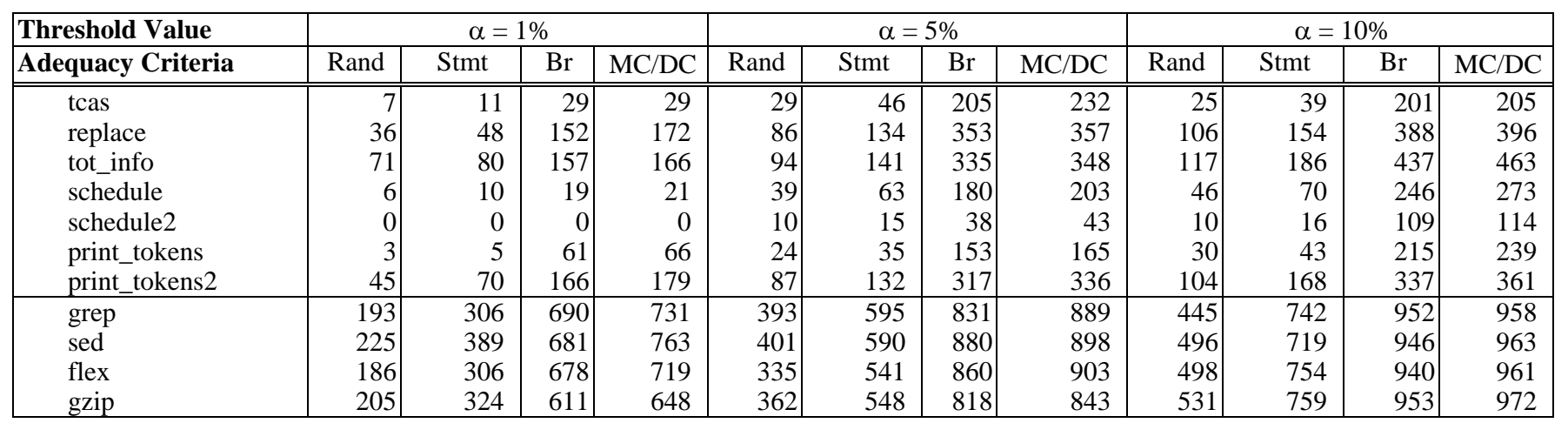

Table 8 Standard deviations of the numbers of effective test suites for SBI.

\begin{tabular}{|c|c|c|c|c|c|c|c|c|c|c|c|c|}
\hline Threshold Value & \multicolumn{4}{|c|}{$\alpha=1 \%$} & \multicolumn{4}{|c|}{$\alpha=5 \%$} & \multicolumn{4}{|c|}{$\alpha=10 \%$} \\
\hline Adequacy Criteria & Rand & Stmt & $\mathrm{Br}$ & $\mathrm{MC} / \mathrm{DC}$ & Rand & Stmt & $\mathrm{Br}$ & $\mathrm{MC} / \mathrm{DC}$ & Rand & Stmt & $\mathrm{Br}$ & $\mathrm{MC} / \mathrm{DC}$ \\
\hline tcas & 2 & 2 & 3 & 4 & 5 & 6 & 4 & 7 & 4 & 5 & 7 & 6 \\
\hline replace & 5 & 3 & 6 & 3 & 4 & 5 & 6 & 5 & 6 & 7 & 5 & 6 \\
\hline schedule & 0 & 0 & 0 & 2 & 0 & 0 & 0 & 0 & 3 & 5 & 6 & 3 \\
\hline schedule2 & 0 & 0 & 0 & 0 & 0 & 0 & 0 & 0 & 0 & 2 & 3 & 7 \\
\hline print_tokens & 0 & 0 & 3 & 5 & 4 & 3 & 4 & 6 & 3 & 5 & 4 & 5 \\
\hline sed & 23 & 30 & 22 & 24 & 20 & 18 & 25 & 20 & 16 & 18 & 11 & 20 \\
\hline flex & 13 & 19 & 24 & 20 & 16 & 19 & 20 & 22 & 15 & 20 & 16 & 19 \\
\hline gzip & 22 & 19 & 17 & 18 & 19 & 21 & 22 & 25 & 16 & 11 & 15 & 14 \\
\hline
\end{tabular}


Table 9 Mean numbers of effective test suites for Jaccard.

\begin{tabular}{|c|c|c|c|c|c|c|c|c|c|c|c|c|}
\hline Threshold Value & \multicolumn{4}{|c|}{$\alpha=1 \%$} & \multicolumn{4}{|c|}{$\alpha=5 \%$} & \multicolumn{4}{|c|}{$\alpha=10 \%$} \\
\hline Adequacy Criteria & Rand & Stmt & $\mathrm{Br}$ & $\mathrm{MC} / \mathrm{DC}$ & Rand & Stmt & $\mathrm{Br}$ & $\mathrm{MC} / \mathrm{DC}$ & Rand & Stmt & $\mathrm{Br}$ & $\mathrm{MC} / \mathrm{DC}$ \\
\hline tcas & 6 & 9 & 31 & 38 & 28 & 41 & 235 & 245 & 25 & 37 & 217 & 232 \\
\hline replace & 17 & 24 & 42 & 46 & 25 & 36 & 206 & 215 & 20 & 33 & 207 & 235 \\
\hline tot_info & 35 & 56 & 153 & 162 & 84 & 138 & 354 & 362 & 103 & 154 & 394 & 421 \\
\hline schedule & 51 & 80 & 147 & 155 & 107 & 158 & 338 & 359 & 126 & 186 & 433 & 456 \\
\hline schedule2 & 8 & 13 & 31 & 40 & 43 & 69 & 180 & 192 & 46 & 69 & 256 & 306 \\
\hline print_tokens & 0 & 0 & 0 & 0 & 8 & 11 & 36 & 42 & 7 & 11 & 109 & 153 \\
\hline print_tokens2 & 11 & 16 & 73 & 82 & 20 & 32 & 156 & 181 & 35 & 51 & 216 & 230 \\
\hline grep & 211 & 306 & 693 & 713 & 343 & 512 & 844 & 867 & 474 & 687 & 920 & 970 \\
\hline sed & 272 & 389 & 660 & 695 & 333 & 513 & 799 & 812 & 493 & 747 & 885 & 899 \\
\hline flex & 193 & 306 & 685 & 692 & 352 & 510 & 805 & 822 & 494 & 705 & 903 & 937 \\
\hline gzip & 220 & 324 & 670 & 711 & 369 & 568 & 852 & 890 & 480 & 695 & 915 & 928 \\
\hline
\end{tabular}

Table 10 Standard deviations of the numbers of effective test suites for Jaccard.

\begin{tabular}{|c|c|c|c|c|c|c|c|c|c|c|c|c|}
\hline \multirow{2}{*}{$\begin{array}{l}\text { Threshold Value } \\
\text { Adequacy Criteria }\end{array}$} & \multicolumn{4}{|c|}{$\alpha=1 \%$} & \multicolumn{4}{|c|}{$\alpha=5 \%$} & \multicolumn{4}{|c|}{$\alpha=10 \%$} \\
\hline & Rand & Stmt & $\mathrm{Br}$ & $\mathrm{MC} / \mathrm{DC}$ & Rand & Stmt & $\mathrm{Br}$ & $\mathrm{MC} / \mathrm{DC}$ & Rand & Stmt & $\mathrm{Br}$ & $\mathrm{MC} / \mathrm{DC}$ \\
\hline tcas & 0 & 0 & 2 & 6 & 7 & 6 & 6 & 10 & 2 & 12 & 5 & 8 \\
\hline replace & 4 & 4 & 8 & 6 & 6 & 12 & 8 & 10 & 3 & 10 & 9 & 11 \\
\hline tot_info & 6 & 5 & 7 & 5 & 6 & 8 & 7 & 8 & 7 & 6 & 11 & 14 \\
\hline schedule & 2 & 4 & 5 & 4 & 12 & 11 & 9 & 10 & 8 & 9 & 6 & 7 \\
\hline schedule2 & 5 & 11 & 7 & 6 & 6 & 8 & 7 & 9 & 5 & 9 & 6 & 6 \\
\hline print_tokens & 0 & 0 & 0 & 0 & 0 & 0 & 3 & 2 & 2 & 4 & 10 & 9 \\
\hline print_tokens 2 & 2 & 0 & 4 & 3 & 6 & 3 & 4 & 4 & 12 & 10 & 9 & 8 \\
\hline grep & 14 & 13 & 9 & 11 & 10 & 16 & 12 & 26 & 21 & 11 & 13 & 18 \\
\hline sed & 16 & 17 & 21 & 23 & 19 & 25 & 13 & 27 & 24 & 8 & 10 & 5 \\
\hline flex & 12 & 16 & 19 & 15 & 16 & 18 & 22 & 24 & 22 & 15 & 25 & 16 \\
\hline gzip & 11 & 22 & 25 & 22 & 24 & 18 & 25 & 22 & 19 & 20 & 32 & 27 \\
\hline
\end{tabular}

Table 11 Mean numbers of effective test suites for Ochiai.

\begin{tabular}{|c|c|c|c|c|c|c|c|c|c|c|c|c|}
\hline Threshold Value & \multicolumn{4}{|c|}{$\alpha=1 \%$} & \multicolumn{4}{|c|}{$\alpha=5 \%$} & \multicolumn{4}{|c|}{$\alpha=10 \%$} \\
\hline Adequacy Criteria & Rand & Stmt & $\mathrm{Br}$ & $\mathrm{MC} / \mathrm{DC}$ & Rand & Stmt & $\mathrm{Br}$ & $\mathrm{MC} / \mathrm{DC}$ & Rand & Stmt & $\mathrm{Br}$ & $\mathrm{MC} / \mathrm{DC}$ \\
\hline tcas & 10 & 15 & 31 & 36 & 30 & 49 & 198 & 202 & 16 & 26 & 201 & 225 \\
\hline replace & 30 & 46 & 163 & 43 & 82 & 130 & 349 & 380 & 93 & 148 & 388 & 411 \\
\hline tot info & 52 & 76 & 157 & 168 & 92 & 138 & 338 & 352 & 118 & 181 & 448 & 506 \\
\hline schedule & 5 & 8 & 12 & 162 & 41 & 61 & 180 & 202 & 38 & 64 & 243 & 292 \\
\hline schedule2 & 0 & 0 & 0 & 34 & 14 & 20 & 48 & 58 & 6 & 9 & 115 & 121 \\
\hline print_tokens & 6 & 9 & 56 & 0 & 20 & 29 & 160 & 168 & 24 & 39 & 231 & 252 \\
\hline print_tokens2 & 38 & 58 & 168 & 80 & 81 & 123 & 320 & 339 & 112 & 173 & 343 & 377 \\
\hline grep & 208 & 306 & 615 & 783 & 363 & 595 & 842 & 926 & 469 & 722 & 900 & 955 \\
\hline sed & 245 & 389 & 669 & 766 & 373 & 574 & 805 & 894 & 451 & 716 & 925 & 972 \\
\hline flex & 208 & 306 & 666 & 822 & 362 & 517 & 824 & 981 & 427 & 712 & 894 & 912 \\
\hline gzip & 194 & 324 & 698 & 764 & 302 & 503 & 834 & 892 & 461 & 709 & 944 & 988 \\
\hline
\end{tabular}

Table 12 Standard deviations of the numbers of effective test suites for Ochiai.

\begin{tabular}{|c|c|c|c|c|c|c|c|c|c|c|c|c|}
\hline Threshold Value & \multicolumn{4}{|c|}{$\alpha=1 \%$} & \multicolumn{4}{|c|}{$\alpha=5 \%$} & \multicolumn{4}{|c|}{$\alpha=10 \%$} \\
\hline Adequacy Criteria & Rand & Stmt & $\mathrm{Br}$ & $\mathrm{MC} / \mathrm{DC}$ & Rand & Stmt & $\mathrm{Br}$ & $\mathrm{MC} / \mathrm{DC}$ & Rand & Stmt & $\mathrm{Br}$ & $\mathrm{MC} / \mathrm{DC}$ \\
\hline tcas & 3 & 0 & 6 & 9 & 5 & 6 & 10 & 8 & 2 & 2 & 5 & 4 \\
\hline replace & 3 & 2 & 4 & 6 & 7 & 5 & 6 & 10 & 15 & 9 & 17 & 17 \\
\hline tot info & 2 & 3 & 5 & 4 & 8 & 4 & 6 & 10 & 13 & 8 & 5 & 6 \\
\hline schēdule & 0 & 0 & 0 & 0 & 3 & 0 & 4 & 5 & 6 & 4 & 3 & 9 \\
\hline schedule2 & 0 & 0 & 0 & 5 & 6 & 5 & 3 & 9 & 6 & 6 & 2 & 9 \\
\hline print_tokens & 0 & 0 & 5 & 0 & 8 & 0 & 3 & 0 & 0 & 0 & 9 & 3 \\
\hline print_tokens 2 & 14 & 8 & 18 & 6 & 5 & 7 & 6 & 2 & 5 & 3 & 9 & 9 \\
\hline grep & 22 & 19 & 17 & 12 & 14 & 26 & 31 & 3 & 12 & 16 & 15 & 19 \\
\hline sed & 17 & 17 & 21 & 19 & 16 & 29 & 5 & 14 & 15 & 9 & 19 & 23 \\
\hline flex & 28 & 25 & 23 & 16 & 21 & 39 & 15 & 11 & 15 & 19 & 8 & 26 \\
\hline gzip & 9 & 10 & 15 & 34 & 15 & 13 & 19 & 14 & 6 & 18 & 27 & 16 \\
\hline
\end{tabular}


Tarantula, SBI, Jaccard, and Ochiai, respectively. In each table, the first row shows the threshold values used in the experiment. We used three threshold values to determine the effectiveness of fault localization, namely, $1 \%, 5 \%$, and $10 \%$. In other words, if a fault can be located by inspecting less than $1 \%$ (or $5 \%$ or $10 \%$ ) of the suggested list of suspicious statements, we deem the fault localization result to be effective. Based on the threshold values, we obtained three such groups of results. The second row shows four adequacy criteria for each group, namely, Rand for the random strategy, Stmt for statement adequacy, $\mathrm{Br}$ for branch adequacy, and MC/DC for MC/DC adequacy. The remaining rows show the means and standard deviations of the numbers of effective test suites for each program. (The total number of test suites for each faulty version is 1000.)

We studied how different adequacy criteria compare with one another without considering test suite size. We observe from Table 5 that for every subject program and every threshold value, on average, the use of an MC/DCadequate test suite performs consistently better than the use of a branch-adequate test suite, which in turn performs consistently better than the use of a statement-adequate test suite. We further conducted ANOVA analysis between $\mathrm{MC} / \mathrm{DC}$ - and branch-adequate test suites as well as between branch- and statement-adequate test suites to see whether each pair differs significantly. The results give small $p$ values (0.0034 and 0.0049 , respectively), which successfully reject the null hypothesis that there is no difference between each pair of them and confirms our observation at a $5 \%$ significance level.

From Table 6, we observe that the standard deviations of the numbers of effective test suites for different adequacy criteria are comparable to one another. We further conducted hypothesis testing, which produced a large $p$-value (0.26) to confirm our observation.

Moreover, the results from Tables 5 and 6 are consistent with those of the other three fault localization techniques in Tables 7 to 12 . The relative order is also consistent with the subsumption relationships among the three test adequacy criteria, where MC/DC adequacy subsumes branch adequacy, which in turn subsumes statement adequacy (in terms of test coverage requirements). Furthermore, the use of each adequacy criterion in supporting statistical fault localization is more effective than random testing. The result is consistent with our conjecture that the adoption of adequacy criteria is, in general, worthwhile.

We also observe that the probability of obtaining an effective test suite for a UNIX program is significantly higher than that for a program in Siemens suites. This observation is interesting. Future work should study the underlying rationales.

To have a better understanding of the results, Fig. 1 shows all the data points in the experiment, each of which represents the code examination effort to locate a fault (measured by Expense) for program versions of different sizes (measured by lines of code). The $x$-axis shows the lines of code for all the Siemens and UNIX programs while the $y$-axis shows the number of lines in the source code that need to be examined to find a fault. Each dot represents the two values 〈LOC, no. of lines examined $\rangle$ for a specific faulty version, and every regression line represents the impact of program size on the code examination effort for a specific test adequacy criterion.

Moreover, when using a fault localization tool as a debugging aid, developers would expect it to help them focus their attention on as small a suspicious code segment as possible; otherwise, the developers may lose patience and consider abandoning the use of the apparently ineffective tool. We observe that a typical debug window of an IDE (such as an Eclipse IDE or a Visual Studio IDE) may show around 25 lines of code without any scrolling. Using this number as a rough benchmark, we have drawn a solid (light blue) horizontal line in Fig. 1.

Interestingly, we find from Fig. 1 that the most of the dots are distributed above the solid (light blue) line, and all the linear regression lines are also above that line. This indicates that in general, it is more likely that fault localization results based on the integration of the adequacy criteria and the statistical fault localization techniques under study do not help developers locate the fault within one debug window of a practical IDE.

Our analysis above represents the start of a new line of research, and the aim of this analysis is not to fully answer whether existing IDEs can effectively present information on statistical fault localization results to developers. However, the finding does raise interesting questions for future work. Can we design a test adequacy criterion that will likely construct test suites with effective fault localization results fitting into one screen? Alternatively, what kind of information should fit into a debug window to support effective fault localization? Also, what kinds of advances in human-computer interaction techniques (such as interactive presentation) will support effective fault localization of large applications?

Furthermore, we find that the slopes of the regression lines for statement-, branch-, and MC/DC-adequate test suites are around 0.0082, 0.0072, and 0.0059, respectively. For example, the slope for MC/DC is 0.0059, which implies that slightly less than 6 extra lines of code need to be examined for every 1000 LOC increase in program size. Moreover, the comparative slopes of the regression lines indicate that the differences in marginal effects of statement-, branch-, and MC/DC-adequate test suites in supporting effective statistical fault localization are significant. The linear equations for the regression lines can be approximated as $y=0.0082+77, y=0.0072 x+56$, and $y=$ $0.0059 x+48$. All the intercepts are positive numbers and more than 25, which indicate that there are overheads in locating faults even in small programs. Such overheads have typically exceeded the usual size of a 25 -line screen in an IDE.

In the above discussion, we have also shown that adequate test suites outperform random test suites. Taking these two points into consideration, it appears that the use of the MC/DC adequacy criterion is the most promising choice 


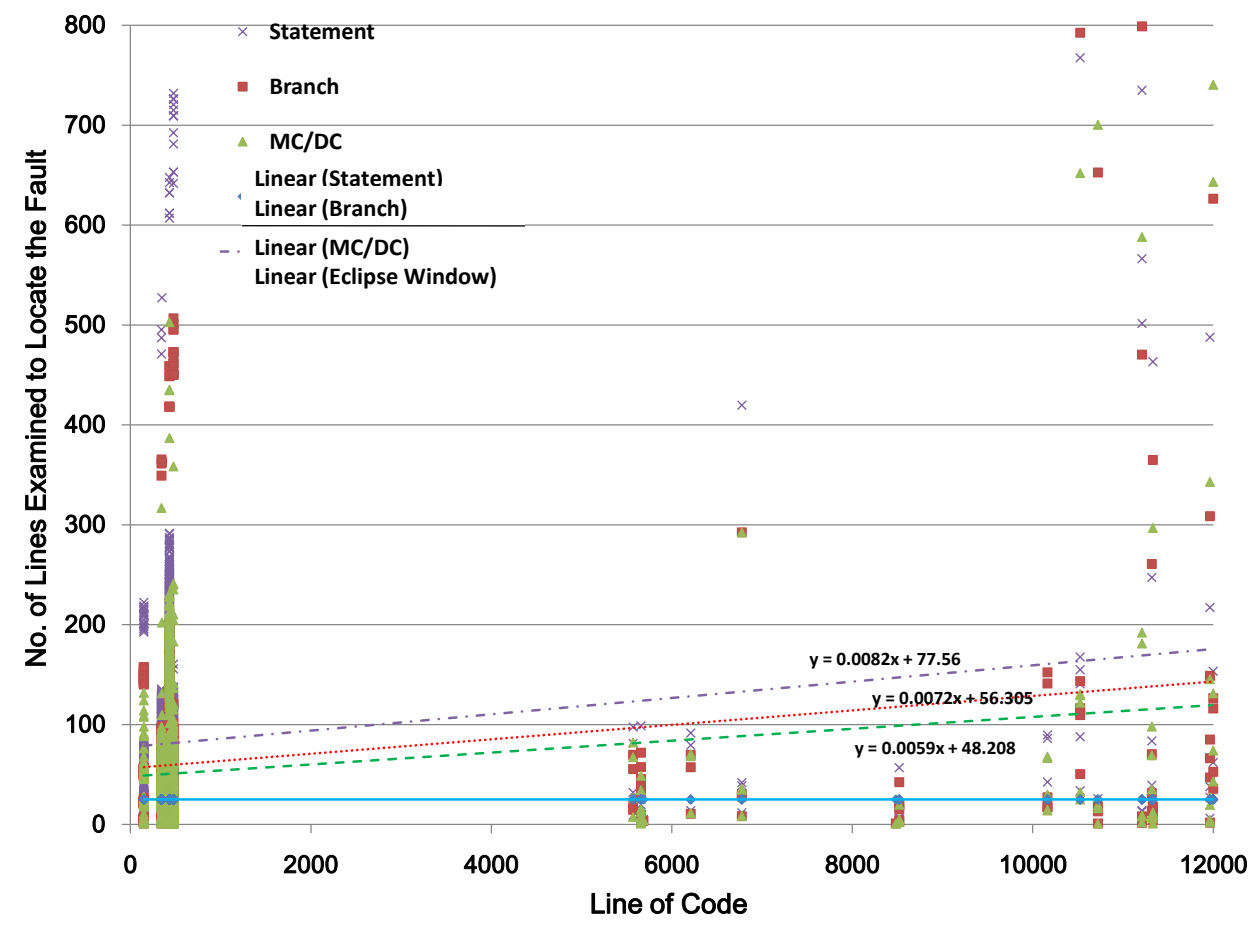

Fig. 1. Distribution of Expense with respect to size of faulty programs.

The slopes of the regression lines are less than 0.0082, 0.0072, and 0.0059, respectively, indicating that the differences in marginal effects of statement-, branch-, and MC/DC-adequate test suites in supporting effective statistical fault localization are significant. Nonetheless, most data points are above the dotted line, indicating that a typical 25-line screen in an IDE may be ineffective in displaying the code that includes the faults.

in addressing the precision and scalability challenges in the integration issue, which we will further verify in the next section.

\subsubsection{Answering $R Q 2$}

From RQ1, we know that using a MC/DC-adequate test suite seems better than using a branch-adequate test suite, which is in turn better than using a statement-adequate test suite. However, a stronger adequacy criterion (higher in the subsumption hierarchy) is usually associated with a larger test suite. Simply using more test cases alone can, on average, provide more information for fault localization. Thus, both the adequacy criterion and the test suite size may have influences on the effectiveness of statistical fault localization. We used a 2-dimensional plot of Expense with respect to test suite size for different adequacy criteria to see if one adequacy criterion is clearly a dominator of another, as shown in Section 3.6.2.1. To compare different test adequacy criteria more fairly, it is necessary to control the impact due to test suite size, which is further presented in Section 3.6.2.1. In addition, different adequacy criteria usually incur different test suite generation costs. Thus, we also measured the test suite generation cost for different adequacy criteria. We will discuss the cost-effectiveness of the studied adequacy criteria in Section 3.6.2.2.

\subsubsection{Adequacy Criteria vs. Suite Size}

Because both test suite size and adequacy criterion are possible factors affecting the fault localization effectiveness of the test suites, we want to first analyze them by drawing 2-dimensional plots of Expense with respect to test suite size for the different adequacy criteria in Figs. 2 and 3, to see whether one adequacy criterion is clearly a dominator of another.

In Fig. 2, the $x$-axis is the test suite size, the $y$-axis is the Expense values of various test suites, and the three trend lines show the Expense values for statement-, branch-, and $\mathrm{MC} / \mathrm{DC}$-adequacy criteria with respect to test suite size over all programs, faulty versions, and fault localization techniques. Fig. 3 shows the same trend lines as those of Fig. 2, except that it shows the results for Siemens and UNIX programs on each fault localization technique separately.

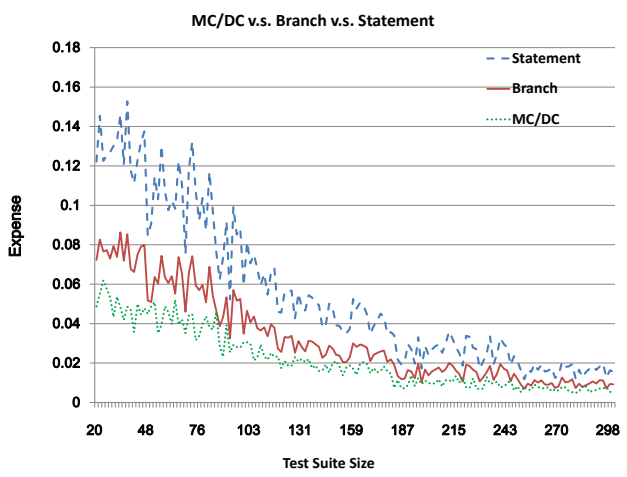

Fig. 2. Impacts of adequacy criteria and suite size on Expense. 


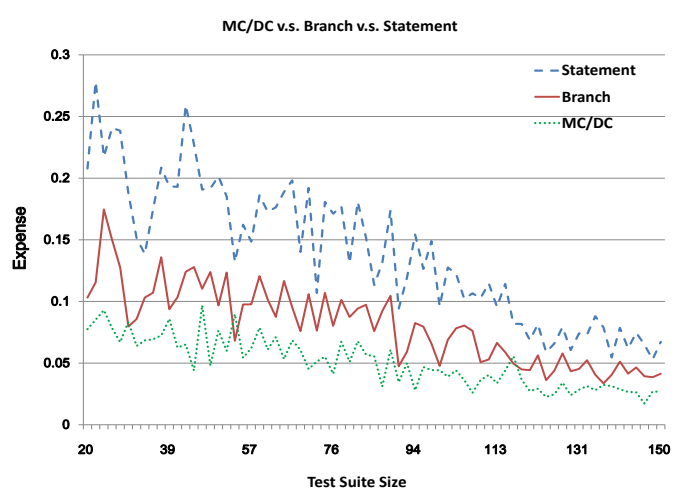

Siemens-Tarantula

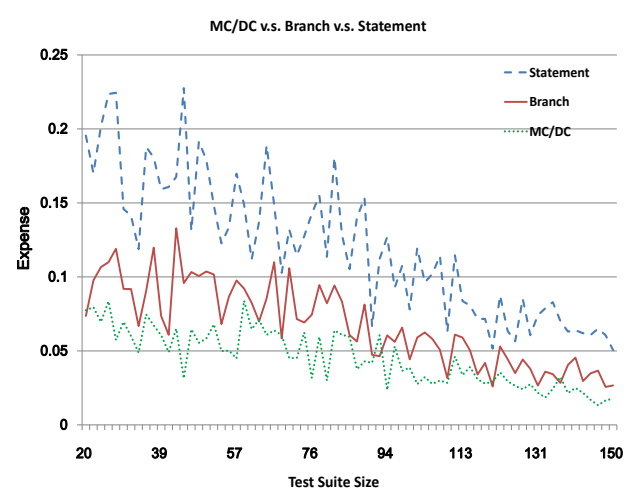

Siemens-SBI

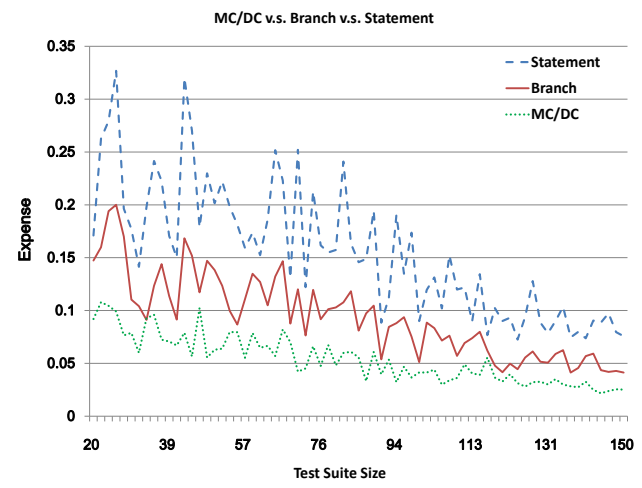

Siemens-Jaccard

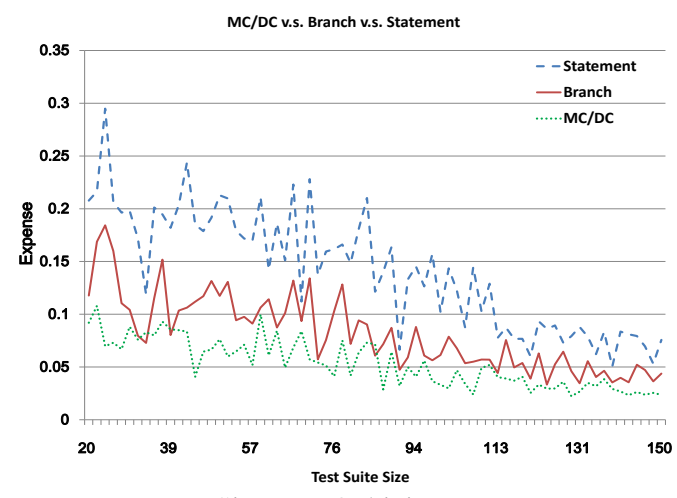

Siemens-Ochiai

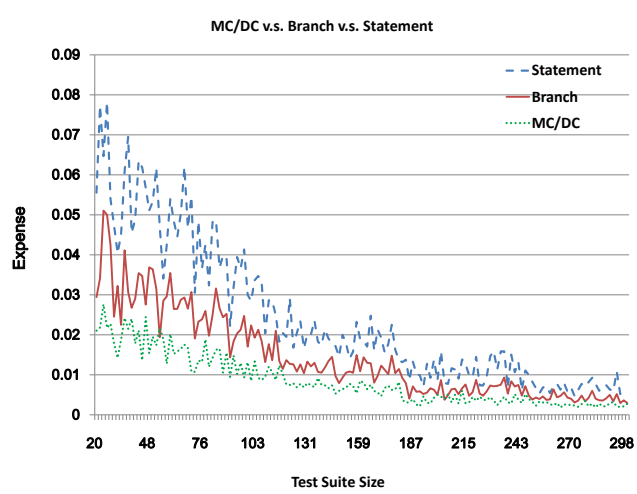

UNIX-Tarantula

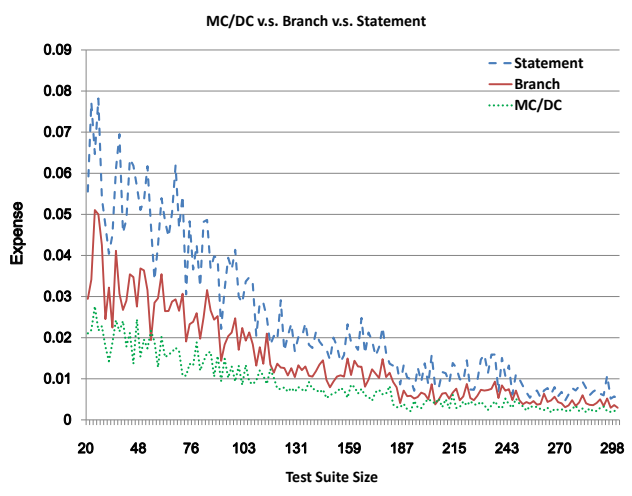

UNIX-SBI

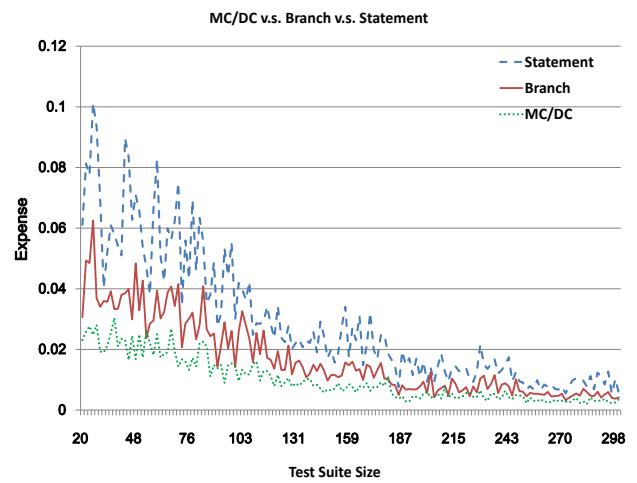

UNIX-Jaccard

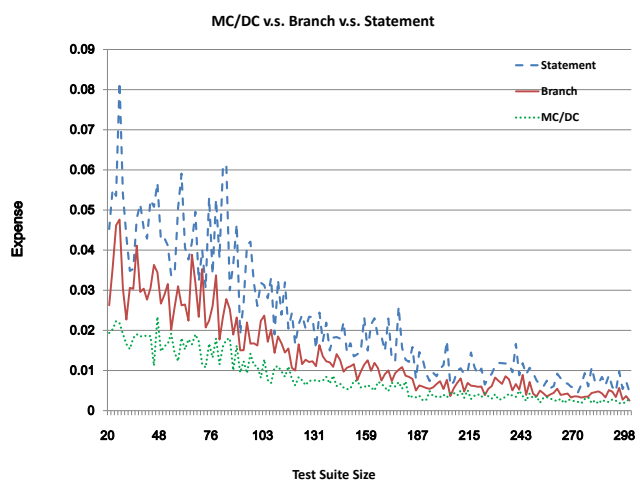

UNIX-Ochiai

Fig. 3. Impacts of adequacy criteria and suite size on Expense for different fault localization techniques. 
If we fix the adequacy criterion, we can also see the trend that the Expense value tends to decrease gradually with larger test suite sizes. This finding affirms previous observations in the literature that larger test suite sizes usually incur better fault localization effectiveness. At the same time, Fig. 3 clearly shows that the trend is not smooth - along part of the curves, some smaller test suites can be more effective than larger ones in terms of Expense, with a difference of as much as 30 test cases in the test suite sizes.

We can see clearly from Figs 2 and 3 that for a fixed test suite size, test suites satisfying the MC/DC-adequacy criterion have consistently lower Expense values than those satisfying the branch-adequacy criterion, which in turn has consistently lower Expense values than those satisfying the statement-adequacy criterion. If we fix the Expense value, we can see that MC/DC-adequate test suites, in fact, requires fewer test cases than branch-adequate test suites, which in turn requires fewer test cases than statementadequate test suites. This clearly shows that statement adequacy, branch adequacy, and MC/DC adequacy are increasingly good at supporting effective fault localization.

\subsubsection{Comparison of Fault Localization Effectiveness}

Tables 13 to 16 compare three pairs of strategies (random vs. statement, statement vs. branch, and branch vs. MC/DC) over all faulty versions for the Siemens and UNIX programs on Tarantula, SBI, Jaccard, and Ochiai, respectively. We summarize and compare what percentage of the adequate test suite for one criterion was better or worse than another in terms of SavingRate. The first row lists the pairs of adequacy criteria to be compared in the experiment. The second row shows the statistics to be compared for each group, namely, Rand for the random strategy, Stmt for statement adequacy, $\mathrm{Br}$ for branch adequacy, and MC/DC for MC/DC adequacy. The remaining rows show the values of the statistics (what percentage of one adequate test suite is better or worse than the other) across all the test suites for every program. For example, the row for tcas shows that among all the test suites for all the faulty versions of tcas, $89 \%$ of the statement-adequate test suites have better fault localization results than random test suites, $78 \%$ of the branch-adequate test suites have better fault localization results than statement-adequate test suites, and $80 \%$ of the MC/DC-adequate test suites have better fault localization results than branch-adequate test suites.

We first studied the comparison results for the three pairs of adequacy criteria in supporting statistical fault localization, considering random as a pseudo-criterion. From Tables 13 to 16, we observe that statement adequacy consistently performs better than the random strategy in terms of SavingRate across all programs and all fault localization techniques. Similarly, we also observe that MC/DC adequacy (or branch adequacy, respectively) consistently performs better than branch adequacy (or statement adequacy, respectively) in terms of SavingRate across all programs and all fault localization techniques.

Table 13. Comparison of SavingRate for different adequacy criteria on Tarantula.

\begin{tabular}{|l|c|c|c|c|c|c|}
\hline \multicolumn{1}{|c|}{ Subject } & \multicolumn{2}{c|}{ Random vs. Statement (\%) } & \multicolumn{2}{c|}{ Statement vs. Branch (\%) } & \multicolumn{2}{c|}{ Branch vs. MC/DC (\%) } \\
\hline & Rand $\geq$ Stmt & Rand < Stmt & Stmt $\geq \mathbf{B r}$ & Stmt < Br & Br $\geq$ MC/DC & Br < MC/DC \\
\hline tcas & 11 & 89 & 22 & 78 & 20 & 80 \\
\hline replace & 9 & 91 & 10 & 90 & 35 & 65 \\
\hline tot_info & 18 & 82 & 25 & 75 & 21 & 79 \\
\hline schedule & 18 & 82 & 17 & 83 & 11 & 89 \\
\hline schedule2 & 15 & 85 & 22 & 78 & 23 & 77 \\
\hline print_tokens & 9 & 91 & 20 & 80 & 24 & 76 \\
\hline print_tokens2 & 6 & 94 & 23 & 77 & 35 & 65 \\
\hline grep & 17 & 83 & 20 & 80 & 32 & 68 \\
\hline sed & 9 & 91 & 16 & 84 & 34 & 66 \\
\hline flex & 6 & 94 & 14 & 86 & 16 & 84 \\
\hline gzip & 5 & 95 & 16 & 84 & 31 & 69 \\
\hline
\end{tabular}

Table 14 Comparison of SavingRate for different adequacy criteria on SBI.

\begin{tabular}{|l|c|c|c|c|c|c|}
\hline \multirow{2}{*}{ Subject } & \multicolumn{2}{|c|}{ Random vs. Statement (\%) } & \multicolumn{2}{c|}{ Statement vs. Branch (\%) } & \multicolumn{2}{c|}{ Branch vs. MC/DC (\%) } \\
\cline { 2 - 7 } & Rand $\geq$ Stmt & Rand < Stmt & Stmt $\geq \mathbf{B r}$ & Stmt < Br & Br $\geq$ MC/DC & Br < MC/DC \\
\hline tcas & 19 & 81 & 24 & 76 & 34 & 66 \\
\hline replace & 8 & 92 & 23 & 77 & 13 & 87 \\
\hline tot_info & 7 & 93 & 10 & 90 & 21 & 79 \\
\hline schedule & 14 & 86 & 23 & 77 & 31 & 69 \\
\hline schedule2 & 19 & 81 & 18 & 82 & 27 & 73 \\
\hline print_tokens & 19 & 81 & 14 & 86 & 35 & 65 \\
\hline print_tokens2 & 17 & 83 & 17 & 83 & 20 & 80 \\
\hline grep & 6 & 94 & 19 & 81 & 18 & 82 \\
\hline sed & 7 & 93 & 12 & 88 & 22 & 78 \\
\hline flex & 20 & 80 & 14 & 86 & 27 & 73 \\
\hline gzip & 18 & 82 & 24 & 76 & 26 & 74 \\
\hline
\end{tabular}


Table 15 Comparison of SavingRate for different adequacy criteria on Jaccard.

\begin{tabular}{|l|c|c|c|c|c|c|}
\hline \multirow{2}{*}{ Subject } & \multicolumn{2}{|c|}{ Random vs. Statement (\%) } & \multicolumn{2}{c|}{ Statement vs. Branch (\%) } & \multicolumn{2}{c|}{ Branch vs. MC/DC (\%) } \\
\cline { 2 - 7 } & Rand $\geq$ Stmt & Rand < Stmt & Stmt $\geq \mathbf{B r}$ & Stmt $<$ Br & Br $\geq$ MC/DC & Br $<$ MC/DC \\
\hline tcas & 16 & 84 & 20 & 80 & 13 & 87 \\
\hline replace & 17 & 83 & 24 & 76 & 32 & 68 \\
\hline tot_info & 15 & 85 & 24 & 76 & 16 & 84 \\
\hline schedule & 11 & 89 & 10 & 90 & 13 & 87 \\
\hline schedule2 & 9 & 91 & 17 & 83 & 35 & 65 \\
\hline print_tokens & 19 & 81 & 23 & 77 & 14 & 86 \\
\hline print_tokens2 & 6 & 94 & 25 & 75 & 15 & 85 \\
\hline grep & 18 & 82 & 21 & 79 & 14 & 86 \\
\hline sed & 14 & 86 & 16 & 84 & 15 & 85 \\
\hline flex & 20 & 80 & 22 & 78 & 21 & 79 \\
\hline gzip & 16 & 84 & 14 & 86 & 11 & 89 \\
\hline
\end{tabular}

Table 16 Comparison of SavingRate for different adequacy criteria on Ochiai.

\begin{tabular}{|l|c|c|c|c|c|c|}
\hline \multirow{2}{*}{ Subject } & \multicolumn{2}{|c|}{ Random vs. Statement (\%) } & \multicolumn{2}{c|}{ Statement vs. Branch (\%) } & \multicolumn{2}{c|}{ Branch vs. MC/DC (\%) } \\
\cline { 2 - 7 } & Rand $\mathbf{\text { Stmt }}$ & Rand < Stmt & Stmt $\geq \mathbf{B r}$ & $\mathbf{S t m t}<\mathbf{B r}$ & $\mathbf{B r} \geq \mathbf{M C / D C}$ & $\mathbf{B r}<\mathbf{M C / D C}$ \\
\hline tcas & 6 & 94 & 25 & 75 & 29 & 71 \\
\hline replace & 10 & 90 & 10 & 90 & 12 & 88 \\
\hline tot_info & 13 & 87 & 25 & 75 & 30 & 70 \\
\hline schedule & 14 & 86 & 14 & 86 & 33 & 67 \\
\hline schedule2 & 16 & 84 & 19 & 81 & 29 & 71 \\
\hline print_tokens & 20 & 80 & 25 & 75 & 27 & 73 \\
\hline print_tokens2 & 12 & 88 & 24 & 76 & 28 & 88 \\
\hline grep & 19 & 81 & 10 & 90 & 12 & 72 \\
\hline sed & 11 & 89 & 19 & 81 & 28 & 69 \\
\hline flex & 16 & 84 & 16 & 84 & 31 & 87 \\
\hline gzip & 17 & 83 & 18 & 82 & 13 & \\
\hline
\end{tabular}

Our observed results are consistent with our conjecture that among the adequacy criteria studied, the stronger the criterion (that is, subsuming others), the more effective it is for testing and debugging.

\subsubsection{Discussion on cost-effectiveness}

Different adequate test suites usually incur different test suite generation costs. In this section, we first measure the cost to select test cases from a test pool to generate an adequate test suite with respect to a test adequacy criterion, and then discuss the cost-effectiveness of different adequacy criteria. As discussed in previous sections, to generate statement-, branch-, and MC/DC-adequate test suites, we iteratively selected test cases from the test pool and added them into the test suite if the coverage achieved by the constructed test suite could be improved in terms of the criterion. The number of test cases within the adequate test suites used in our experiments varied from around 20 to 300 for different criteria and programs. The size of the random test suite ranged from $A / 2$ to $A \times 2$, where $A$ is the average size of the branch adequacy suites for the same program. When constructing the test suites in our experiment, we recorded and calculated the means and standard deviations of the test suite generation times (in ms) for different test adequacy criteria, as shown in Table 17.

We can see that the time taken to generate a random test suite is negligible. When comparing the mean test suite generation time for statement-, branch-, and MC/DCadequate test suites, we can find that generating branchadequate test suites incurs the smallest cost. Generating the MC/DC-adequate test suite incurs higher cost than generating statement-adequate test suite, which in turn involves higher cost than branch adequacy. We find the standard deviations of the time costs for generating different adequate test suites to be very close. Although different adequacy criteria involve different costs, the absolute time cost of the most expensive criterion (MC/DC) is not high for our subject programs. On average, our tool will take less than 23 seconds to generate a MC/DC-adequate test suites from the test pool. On the other hand, adopting stronger criteria like $\mathrm{MC} / \mathrm{DC}$ will provide more precise debugging aid to save human debugging time, which can be very long. In general, trading affordable machine execution time for human code inspection time during debugging is quite worthwhile. This is because the former can run in the background while the latter is usually on the critical path in software development.

Table 17 Means and standard deviations of test suite generation times for different adequacy criteria.

\begin{tabular}{|l|c|c|c|c|}
\hline Adequacy Criteria & Random & Statement & Branch & MC/DC \\
\hline Mean (ms) & 0.01 & 12988 & 8267 & 22568 \\
\hline Standard deviation & $<0.01$ & 3550 & 2619 & 3815 \\
\hline
\end{tabular}


In general, a test case may be generated automatically, semi-automatically, or manually. Our result is applicable when comparing test suite construction costs under the automatic scenario. A further extension of the present study to cover the cost-effectiveness of testing-debugging integration for manual or semi-automatic test case constructions can be useful. To the best of our knowledge, the vast majority of existing research work on test adequacy criteria, test case prioritization, and statistical fault localizations do not deal with the human aspects of computing. They are certainly interesting to explore. One consideration for such explorations is that the experimentation requires controlling the content of the test pool among different test adequacy criteria to be compatible (if not identical) even when heterogeneous manual processes are involved. Furthermore, convincing show cases to demonstrate an effective integration between automatic test case generation and our work will also be crucial to make the research results more transferrable to the industry.

\subsubsection{Answering RQ3}

To answer RQ3, we conducted postmortem analysis on the integration results. Owing to the large number of possible standards to determine whether an integration is effective, we used three different threshold values of Expense, namely, $\alpha=0.01,0.05$, and 0.10, as the criteria to deem a test suite to be effective. They represent the cases that developers need to examine up to $1 \%, 5 \%$, and $10 \%$ of the code in order to locate the faults. They were used in the conference version of this paper. In the present study, we analyze RQ3 based on the two best adequacy criteria from RQ1 and RQ2: branch adequacy and MC/DC adequacy. When studying RQ3, we present the overall results of all prioritization techniques rather than showing each of them separately. We want to explore whether the adoption of test case prioritization techniques in general can be helpful for statistical fault localization.

\subsubsection{Siemens programs with branch-adequate test suites}

Figs. 4(a), (c), and (e) show the results of using branchadequate test suites on Siemens programs. In each of these subfigures, the $x$-axis represents different percentages of a test suite used for fault localization while the $y$-axis represents the FLSP values (by examining the percentage of code up to the threshold value) for applying a test case prioritization technique before locating faults.

We observe from Fig. 4(a) that, by inspecting the top 1\% of the ranked list of statements, the median FLSP value of a test suite is $8 \%$ if we prioritize and run the top $10 \%$ of a test suite for fault localization, which is very low. Even if we increase the percentage of test suite to $100 \%$, the median of the percentages of effective test suites is still less than $14 \%$. The result indicates that it is still unlikely to locate the fault in the few (say, 1 to 5) top-ranked statements.

From Fig. 4(a), (c), and (e), we observe that if a higher percentage of an original test suite is used for fault localization, the percentage of effective test suites increases. However, the increase is gradually less intense when the percentage of the test suite used reaches $60 \%$. In particular, given a code inspection range of $1 \%$, the use of $60 \%$ of the prioritized test cases for the fault localization already achieves a FLSP value of 13\%, whereas the use of all the remaining $40 \%$ of test cases will only increase the percentage value up to $14 \%$. We observe similar trends for code inspection ranges of 5\% and 10\% in Fig. 4(c) and (e), respectively.

We also performed an ANOVA analysis to compare the mean FLSPs. The small $p$-value of 0.0032 consistently rejects the null hypothesis that the use of different percentages (namely, $10 \%, 20 \%, \ldots, 100 \%$ ) of the same ordered test suites has the same FLSP values, at a significance level of $5 \%$.

Fig. 4(a), (c), and (e) only show that there are differences in effectiveness when using various percentages of test suites for statistical fault localization, but they cannot tell whether they differ significantly. To see what percentage of test suites differ from one another in terms of FLSP, we further performed the multiple comparison procedure to find how different percentages of test suites differ significantly from one another at a significance level of 5\%. Figs. 4(b), (d), and (f) show the results. The solid lines not intersected by the two vertical lines represent the percentages of test suites whose means differ significantly from the use of $60 \%$ of the suite for fault localization, while the gray lines represent the percentages of test suites comparable to the use of $60 \%$ of the suites for fault localization.

From Figs. 4(b) and (d), we find that executing $60 \%$ of a test suite has no significant difference from executing the entire test suite. If we relax the code examination range to $10 \%$ for the Siemens suite, as shown in Fig. 4(f), there will be a significant difference. It indicates that developers should estimate the amount of code they can afford to examine so that a test case prioritization technique can use it as a reference to determine the proportion of test suites to be executed.

\subsubsection{UNIX utility programs with branch-adequate test suites}

We also conducted the same postmortem analysis on the integration study for UNIX programs with branch-adequate test suites as we have presented in Section 3.5.3.1. Fig. 5(a), (c), and (e) show the results.

We observe from Fig. 5(a) that, by inspecting the top 1\% of the ranked list of statements, the median FLSP value is $47 \%$ if we prioritize and execute the top $10 \%$ of a test suite for fault localization, which is much higher than that for the Siemens programs. Even if we increase the percentage of test suite to $100 \%$, the median FLSP value is still under $65 \%$. Although developers are willing to examine up to 5\% (or 10\%, respectively) of the code, Fig. 5(c) (or Fig. 5(e), respectively) still shows that there is less than $65 \%$ (or $73 \%$, respectively) of chance that the top $10 \%$ of test cases can assist them in locating faults effectively. The results show that developers should not greedily start fault localization based on a small percentage (10\% in the above discussion) of the whole test suite.

The data show that there can be at least two strategies to address this problem. First, we observe across Fig. 5(a), (c), 
and (e) that since the corresponding bars among the three plots increase in terms of their $y$-values, it may be worthwhile to put in more effort in examining the code. Second, on each plot in Fig. 5(a), (c), and (e), when a higher percentage of an original test suite is used for fault localization, the percentage of effective test suite increases remarkably. The results suggest that, if the preferred code examination range is fixed, the use of a higher percentage of

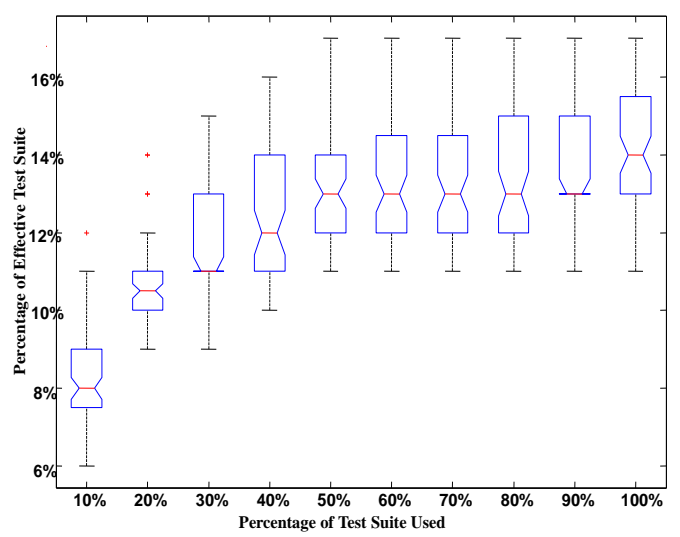

(a) Expense $<1 \%$

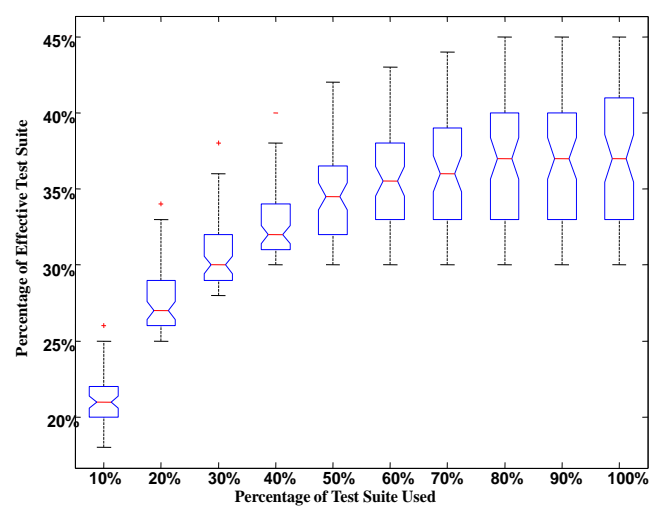

(c) Expense $<5 \%$

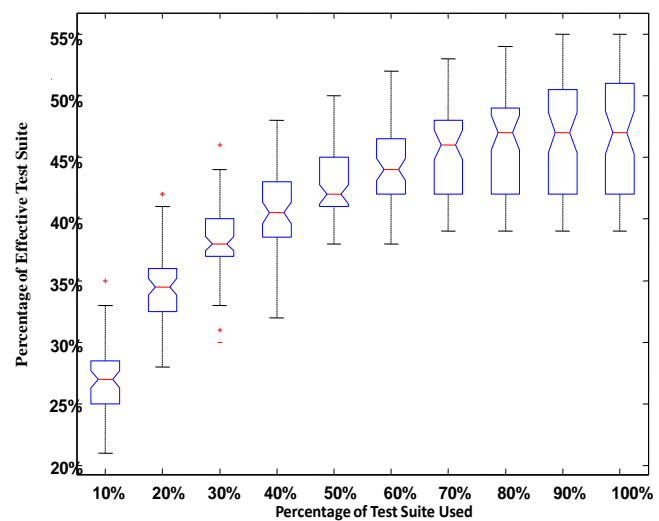

(e) Expense $<10 \%$ test cases can be a good choice. It seems to us that this second strategy provides hints to answer the follow-up question in RQ1 that, in order to fit the code into one codeview screen, the use of a smaller adequate test suite for such testing-debugging integration may be a viable research direction. (However, the study on this aspect is outside the scope of this paper.)

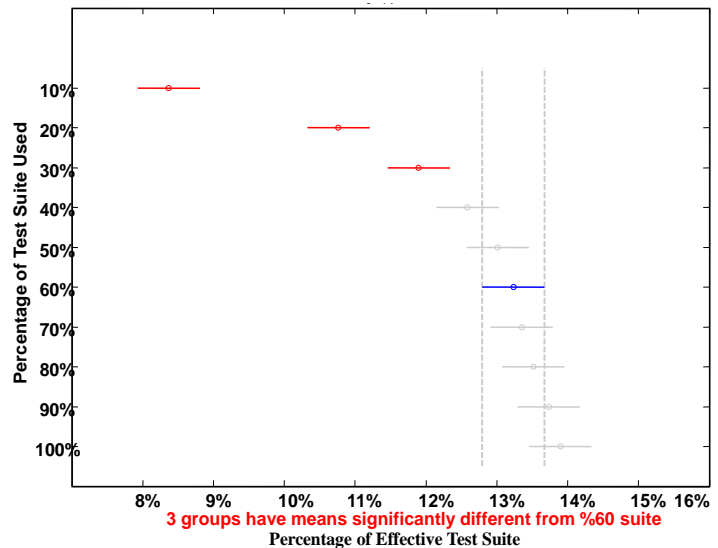

(b) Expense $<1 \%$

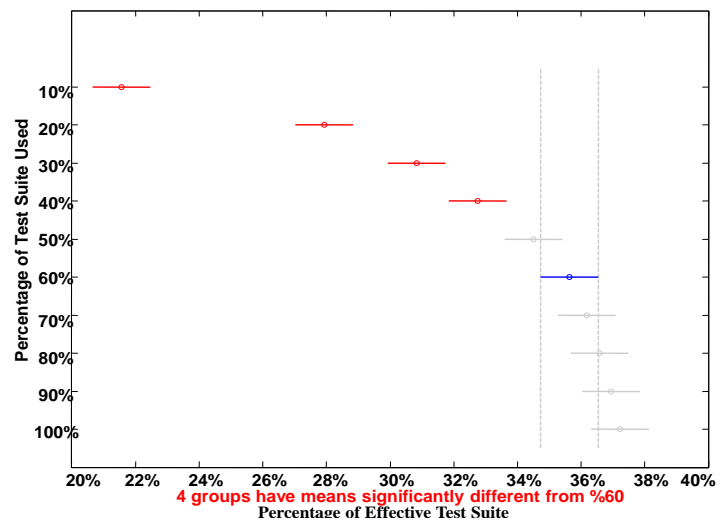

(d) Expense $<5 \%$

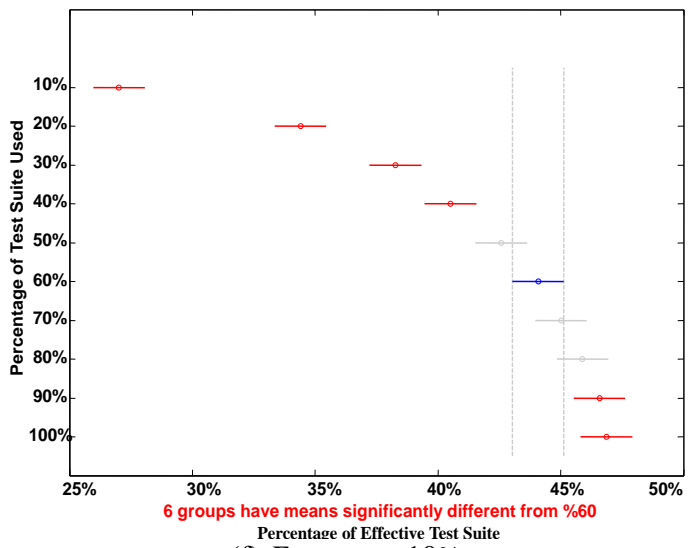

(f) Expense $<10 \%$

Fig. 4. The chance of test case prioritization techniques supporting effective fault localization using branch-adequate test suite for Siemens programs. 


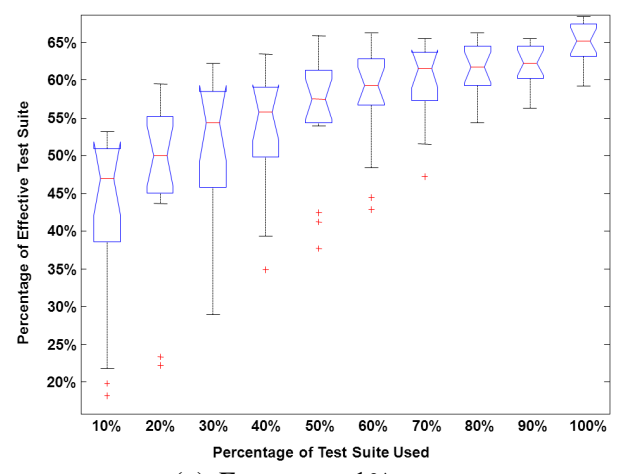

(a) Expense $<1 \%$

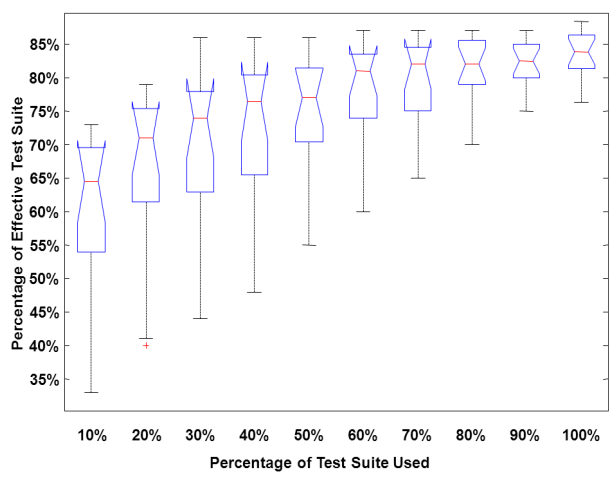

(c) Expense $<5 \%$

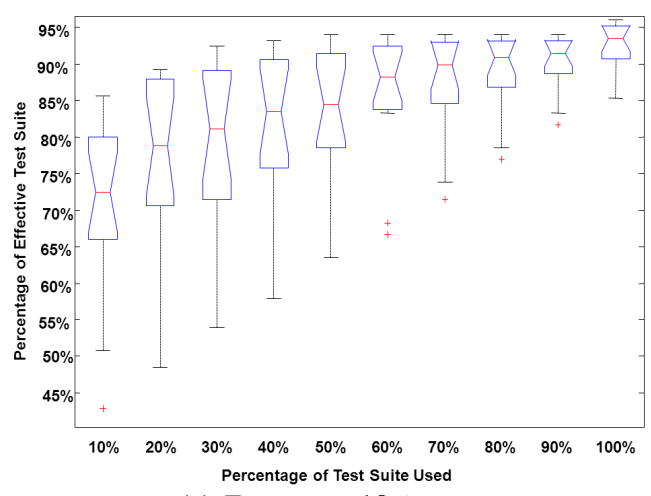

(e) Expense $<10 \%$

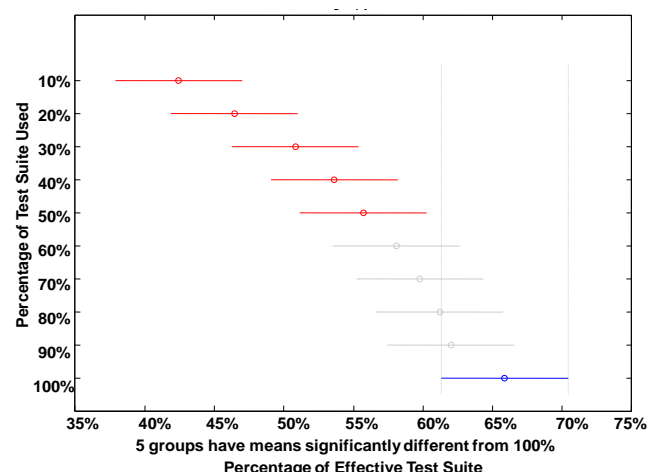

(b) Expense $<1 \%$

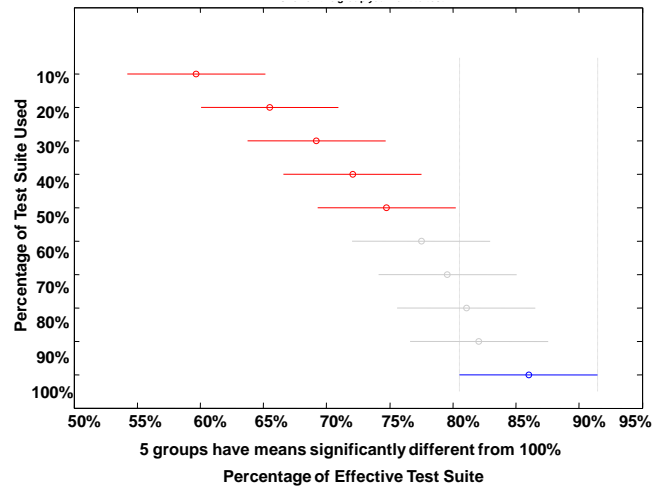

(d) Expense $<5 \%$

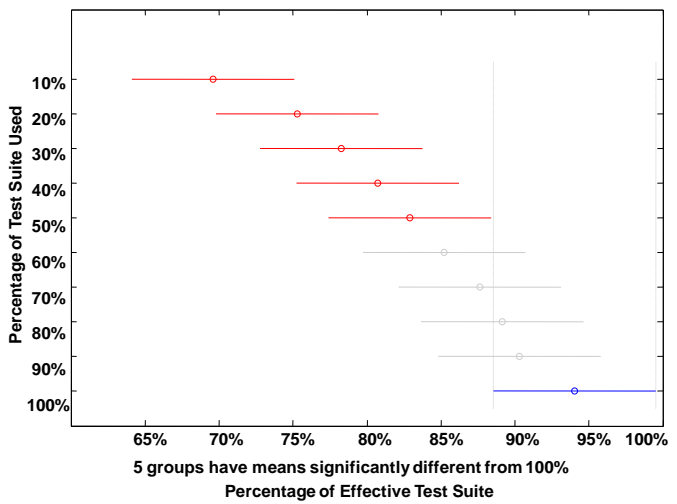

(f) Expense $<10 \%$

Fig. 5. The chance of test case prioritization techniques supporting effective fault localization using branch-adequate test suites for UNIX programs.

We perform ANOVA analysis to compare the mean FLSPs. The small $p$-value of 0.0142 rejects the null hypothesis (at a significance level of 5\%) that the use of different percentages of test suites generates identical FLSP values. We further conduct the multiple comparisons procedure to find how different percentages of the same ordered test suites differ significantly from one another at a significance level of 5\%. Fig. 5(b), (d), and (f) show the results. The solid lines not cut by the two vertical lines represent those percentages of test suites whose mean values differ significantly from the use of $100 \%$ of the suite for fault localization, while the gray lines represent those proportions of test suites whose effectiveness is comparable to the use of $100 \%$ of the suites for fault localization.

We observe from Fig. 5(b) that only when executing more than $60 \%$ of a test suite will there be no significant difference from executing the entire test suite in terms of FLSP. If we relax the code examination range to $5 \%$ and $10 \%$ of the code as shown in Fig. 5(d) and (f), respectively, we still have the same results. It indicates that, for UNIX programs, around $60 \%$ of the test suite should be used to obtain fault localization effectiveness comparable to the use of the whole test suite. The results indicate that, if smaller test suites are used, the fault localization effectiveness is extremely likely to be decreased. 


\subsubsection{Siemens programs with MC/DC adequacy suites}

Figs. 6(a), (c), and (e) show the corresponding results on the Siemens programs using the MC/DC-adequate test suites. The same procedure as described in Section 3.6.3.1 was used except that we used the MC/DC-adequate test suites rather than the branch-adequate test suites. We observe from Fig. 6(a) that by inspecting the top $1 \%$ of the ranked list of statements, the median FLSP value of a test suite is around $12.8 \%$ if we prioritize and execute the top $10 \%$ of the test suite for fault localization, which is still low. If we increase the percentage of test suite to $100 \%$, the median percentage of effective test suites is still less than $22 \%$. Similar to the result for branch-adequate test suites, the present result indicates that for the MC/DC-adequate test suites, it is also quite impractical to assume that the faults will be in the few (say, 1 to 5) top-ranked statements.

From Figs. 6(a), (c), and (e), we find that if a higher percentage of an original test suite is used for fault localization, the percentage of effective test suites increases. However, the increase is gradually less noticeable when the percentage of the test suite used reaches $60 \%$. In particular, given a code inspection range of $<1 \%$, the use of $60 \%$ of the prioritized test cases for the fault localization already achieves a FLSP value of $21 \%$, whereas the use of all the remaining $40 \%$ of test cases will increase the percentage to $23 \%$ at most. We observe similar trends for code inspection ranges of $<5 \%$ and $<10 \%$ in Fig. 6(c) and (e), respectively.

We conducted ANOVA analysis to compare the mean FLSPs. The $p$-value 0.021 rejects the null hypothesis that the use of different percentages (namely, $10 \%, 20 \%, \ldots$, $100 \%$ ) of the same ordered test suites has the same FLSP value, at a significance level of 5\%. To see what percentages of test suites differ from one another in terms of FLSP, we further conducted multiple comparisons to find how different percentages of test suites differ significantly from one another, at a significance level of 5\%. Fig. 6(b), (d), and (f) show the results. The solid horizontal lines not intersected by the two vertical lines represent the percentages of test suites whose mean values differ significantly from the use of $60 \%$ of the suite for fault localization, while the gray lines represent the percentages of test suites whose usage is comparable to the use of $60 \%$ of the suites for fault localization. We observe from Figs. 6 (b) and (d) that executing $60 \%$ of a test suite has no significant difference from executing the entire test suite.

If we compare between Figs. 4 and 6, we can find that for both adequacy criteria, they show similar trends as a larger proportion of an adequate test suite is used for statistical fault localization. This implies that for the Siemens programs, around $40 \%$ of test suite can be avoided from execution without significantly compromising fault localization effectiveness.

There are also differences between MC/DC adequacy and branch adequacy in supporting fault localization. Let us compare the corresponding subfigures in Figs. 4 and 6. We find that larger proportions of MC/DC-adequate test suites are more effective in supporting fault localization than those of branch-adequate test suites. Our hypothesis testing confirms that the difference is statistically significant at a level of 5\%. This is consistent with our earlier finding that MC/DC adequacy is stronger than branch adequacy in supporting effective fault localization.

\subsubsection{UNIX utility programs with MC/DC adequacy suites}

We also conducted postmortem analysis on the integration study for the UNIX programs using the MC/DC adequacy suites. Fig. 7(a), (c), and (e) show the corresponding results. The meanings of the $x$ - and $y$-axes are similar to the corresponding subfigures in Fig. 6.

We observe from Fig. 7(a) that by inspecting the top 1\% of the ranked list of statements, the median FLSP value is $54 \%$ if we order a test suite by prioritizing the test cases and execute the top $10 \%$ of them for fault localization, which is much higher than that for the Siemens programs. Even if we increase the percentage of test suite to $100 \%$, the median FLSP value is still under $75 \%$. If we compare the results of Fig. 7 with those of Fig. 5, we find that fault localization effectiveness on the MC/DC-adequate test suites performs consistently better than that on the branch-adequate test suites. We also performed hypothesis testing to confirm that the difference is significant at a 5\% significance level. This echoes our finding in earlier sections that stronger (subsuming) adequacy criteria can support statistical fault localization better than weaker (subsumed) ones.

Similar to the discussions on Fig. 5, we observe from Fig. 7(b) that only when executing more than $70 \%$ of a test suite will there be no significant difference (in terms of FLSP) from executing the entire test suite. If we relax the code examination range to $5 \%$ and $10 \%$ of the code as shown in Fig. 7(d) and (f), we still have similar results (70\% and $60 \%$, respectively). It shows that for the UNIX programs and the MC/DC-adequate test suites, around 60-70\% of a test suite should be used to obtain a fault localization effectiveness comparable to the use of the whole test suite. The results indicate that using test case prioritization is highly recommended in the integration process as it can save as much as $40 \%$ of test case execution without affecting fault localization effectiveness.

We also looked into the test cases of the effective adequate test suites (including statement adequacy, branch adequacy, and MC/DC adequacy). We found that they are effective in locating faults due to several reasons. First, they may cover failure-revealing paths that are relatively difficult to cover in code coverage. Second, these adequate test suites on average have higher failure rates. Third, the branch or MC/DC coverage criterion has relatively more even coverage over all the possible paths, which makes the comparison between pass and fail test cases more precise and significant.

To conclude our finding, we can answer RQ3 that the probability of using a test case prioritization technique to generate effective test suites for statistical fault localization is higher on the UNIX programs than on the Siemens programs. Furthermore, around $70 \%$ of a test suite should be used to retain the fault localization effectiveness of the whole test suite. Finally, we find that applying a stronger adequacy criterion not only indicates better testing effectiveness but also achieves better fault localization support. 


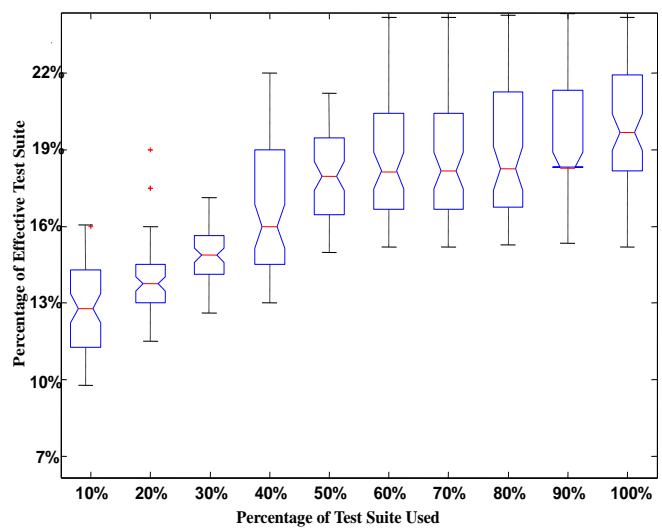

(a) Expense $<1 \%$

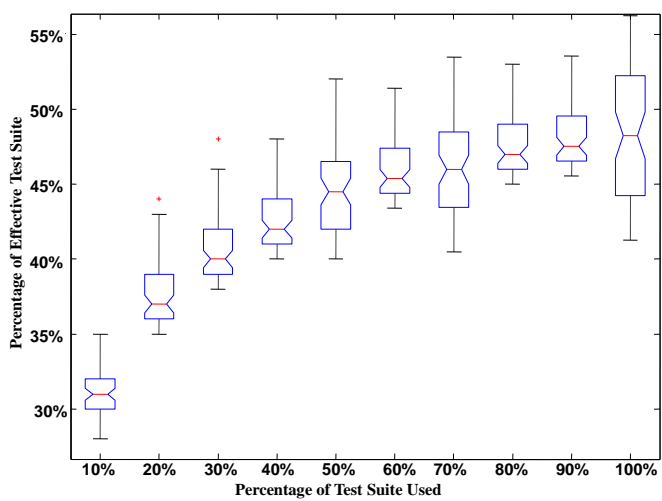

(c) Expense $<5 \%$

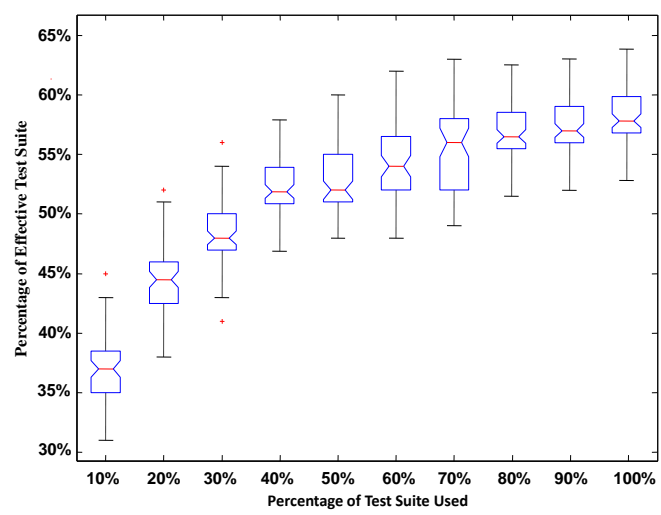

(e) Expense $<10 \%$

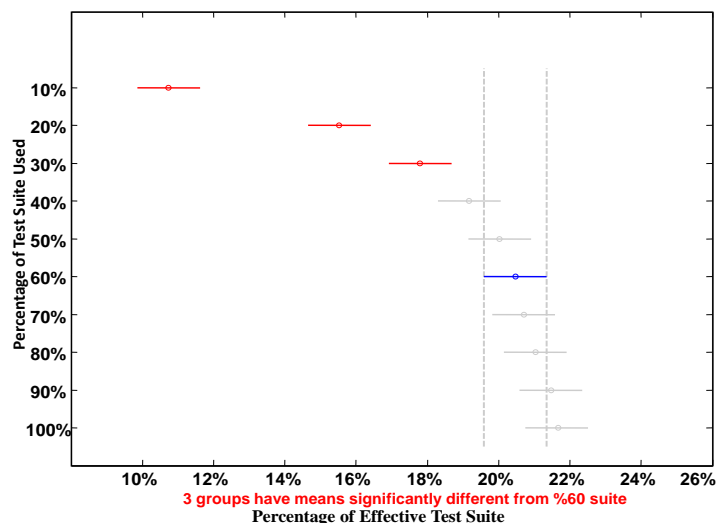

(b) Expense $<1 \%$

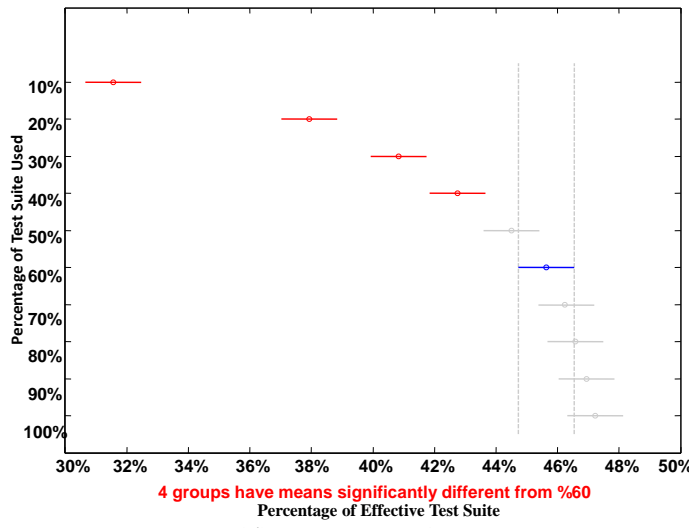

(d) Expense $<5 \%$

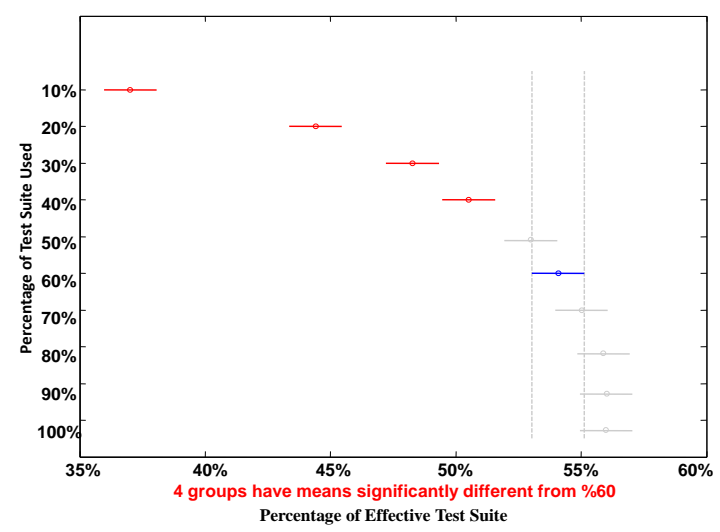

(f) Expense $<10 \%$

Fig. 6. The chance of test case prioritization techniques supporting effective fault localization using MC/DC-adequate test suite for Siemens programs.

\subsection{Threats to validity}

We used seven Siemens programs, four UNIX programs, and their accompanied faulty versions as our subjects. The use of other subject programs may result in different coverage patterns for failed test executions and passed test executions, which may result in different suspiciousness values assigned to the program statements. Although the set of faults cannot represent all possible faults, using them to conduct comparisons among techniques published in peer work is useful for researchers to compare results across different papers and experiments. Moreover, we used the adequate test suites provided by the SIR repository for Siemens programs and generated the branch- and statementadequate test suites for the UNIX programs. We also generated MC/DC-adequate test suites for both Siemens and UNIX programs. The use of other adequate test suites may provide other results. We will leave the analysis and reporting of such test suites as future work. 


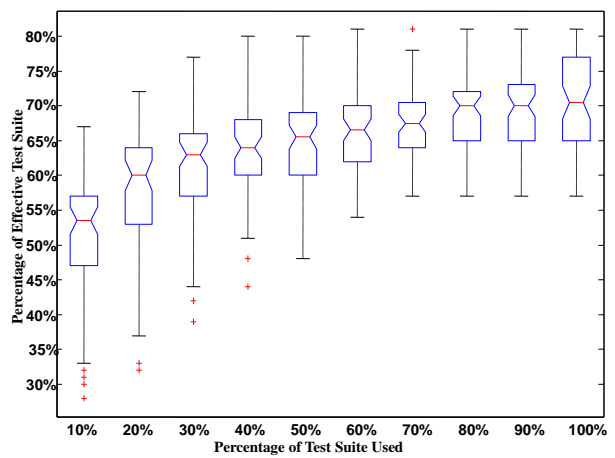

(a) Expense $<1 \%$

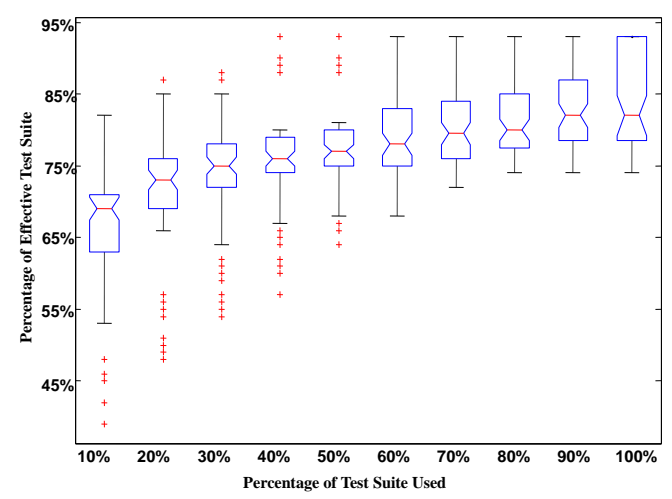

(c) Expense $<5 \%$

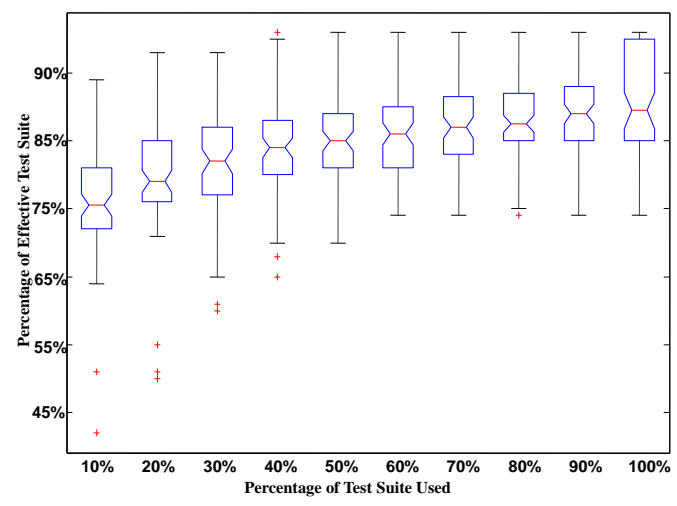

(e) Expense $<10 \%$

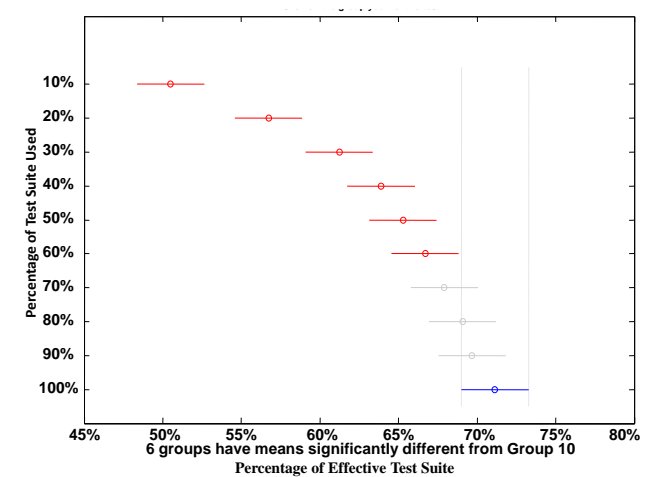

(b) Expense $<1 \%$

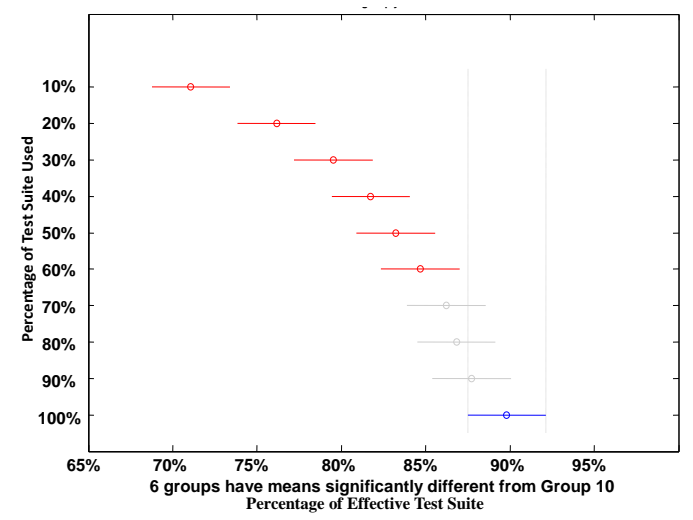

(d) Expense $<5 \%$

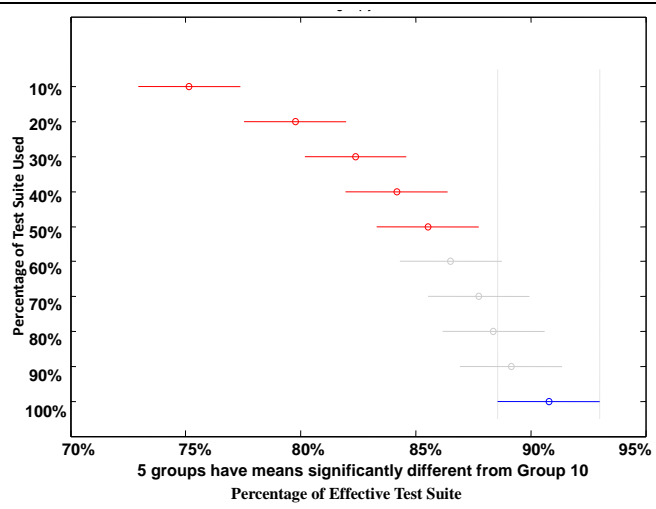

(f) Expense $<10 \%$

Fig. 7. The chance of test case prioritization techniques supporting effective fault localization using MC/DC-adequate test suite for UNIX programs.

In any case, our subjects have been widely used in existing test case prioritization, statistical fault localization, and regression testing research. Furthermore, branch- and MC/DC-adequate test suites have frequently been used in the experiments of testing and debugging papers. We believe that they have used these subjects in their experiments on solid basis with practical considerations. The results of our experiment complement their findings on these artifacts and help comparison across publications.

In this work, we used single fault versions of the subject program to perform the empirical study. On one hand, the single fault assumption is also frequently used in many other empirical studies. On the other hand, we recognize that a program may contain multiple faults in practice. Due to the tremendous scale of our current empirical study, we will leave the study of multi-fault versions as future work.

In our experiment, we excluded some faulty versions and test cases available from SIR. There are several reasons. The foremost reason is that in our experimental framework, we use gcov, a popular and freely available tool, to collect the branch and statement execution profile of each noncrashed execution. For crashed executions, gcov cannot provide coverage data. The techniques in our experiment, however, require coverage data in order to operate. Conse- 
quently, we excluded these test cases from the data analysis. As we have reported, our experimental environment was a UNIX server running Solaris. The $\mathrm{C}$ compiler on the underlying platform was provided by Oracle. Some versions could not be compiled. This was a platform-dependent issue and we removed these versions to reduce their impact.

Another reason for us to exclude some faulty version from the data analysis is that we followed previous papers on test case prioritization to conduct the experiment to exclude any version whose failures can be detected by more than $20 \%$ of the test cases in the test pool. The choice of this threshold value poses a threat to this study. Nonetheless, this practice has been widely used in the test case prioritization experiments. The use of this threshold value facilitates a comparison between this work and existing publications. A way to address this threat could be to conduct a larger experiment to vary this threshold from $0 \%$ to $100 \%$ systematically, and observe the effect. The effort to conduct this experiment and the corresponding data analysis are, however, overwhelming for us. We, therefore, excluded this aspect from our current experiment.

In Tables 5, 7, 9 and 11, the differences between the UNIX programs and the Siemens programs are dramatic. To avoid the internal validity caused by our subject programs, tools and results analysis procedures, we carefully checked and verified them, which confirmed the results. We believe that the program size, faults seeded, as well as other program features may explain these big differences, which we will explore as future work.

Another concern about the study may be the characteristics of the test suites. We used the test suites provide $3 \pi \mathrm{d}$ by SIR. They may not be representative in the sense that some test cases important to statistical fault localization may not be available. On the other hand, test case prioritization and fault localization are becoming mature and hence a common basis for comparison is necessary. To strike a balance between the use of more test suites and the comparability with a large body of published work, we chose the latter option in this study. In RQ1, we had 1000 branch-adequate test suites, 1000 statement-adequate test suites, 1000 MC/DC-adequate test suites, and 1000 random test suites for each subject program. They provided us with sufficient data points to compile statistical results shown in the paper. For RQ2, we would like to highlight that the results were based on one small test pool per subject program. As a result, we should not overly generalize the results. For some subject programs, the requirement of having branch- or MC/DC-adequate test suites may still be too demanding. For instance, almost all the subject programs used in the experiment reported in [5] did not come with test suites that are branch adequate or MC/DC adequate. We will leave this practical consideration as future work.

Another potential threat to validity is that the way we constructed adequate test suites may be different from that used in practice. It might be the case that a test suite constructed to target $\mathrm{MC} / \mathrm{DC}$ has different features than one including test cases sampled from a pool until MC/DC is achieved. The evaluation of the impact of different adequate test suite construction strategy on fault localization effec- tiveness can be left as future work.

In this study, owing to time and resource limitation, we only evaluated random ordering, the coverage-based Greedy, and the white-box $A R T$-based test case prioritization techniques. Although they are among the best general test case prioritization techniques studied in previous work, they have not been optimized. The use of optimized versions or other variants of these strategies as well as the use of other strategies may produce different results.

In drawing a comparison, we used the Expense metric, the FLSP metric, and the SavingRate metric. The use of other metrics may produce different results. The Expense metric has been widely used to evaluate statistical fault localization techniques. It, however, only represents one possible way of how developers may use the ranked list of statements, and it makes an assumption that any fault on each visited statement can be identified correctly with the same amount of effort. The time taken to evaluate such a statement and the precision of the fault identification has not been captured by this metric. The FLSP metric is built on top of the Expense metric. Owing to the limitation of the original metric, the effort to reveal a fault measured by the FLSP metric may not fully reflect the effort of developers to use the generated ranked list of statements to perform debugging. Readers are advised to interpolate the results of the experiment carefully. Finally, SavingRate is only one possible approach to discounting the influence of test suite size. Researchers may adopt other approaches to achieve the same goal.

Furthermore, the adequate test suite construction method for a stronger criterion such as MC/DC can be costly in practice. Our adequate test suite construction approach to selecting from a large test pool may incur less manual effort than that used in practice. Thus, there is a potential tradeoff: adopting a stronger adequacy criterion can improve fault localization precision and save debugging effort while it may also incur higher test suite construction effort. A study of the issue using different adequate test suite construction approaches in the industry can further strengthen the validity of our empirical study, which is left as future work.

We also measured the means and standard deviations of different adequacy criteria. Our results show that different adequacy criteria have significantly different mean values in terms of SavingRate, although the absolute difference seems to be not large. The standard deviations are small. However, when the sizes of regression test suites are large (which is often the case in practice), the absolute difference will become much larger. To strengthen the validity of our results, we carefully verified our results to ensure the statistical difference was not due to large sample size.

\section{Related work}

Previous work has also studied the integration problem between testing and debugging.

Wong and colleagues proposed a technique to integrate test suite minimization and prioritization together [28]. Their heuristics is to select test cases based on the cost per additional coverage. Baudry et al. [4] used a bacteriologic approach to generating test cases in order to maximize the 
number of dynamic basic blocks. In this way, the fault localization techniques can be more effective. $\mathrm{Yu}$ and colleagues [29] explored the impact of using test suite reduction on fault localization. Their results show that test suite reduction does have an impact on fault localization effectiveness. However, test case prioritization differs from test suite reduction techniques in that test case prioritization is more flexible when allocating testing resources. If we use test case prioritization, the resources are used to execute the most important test cases, regardless of the time to stop. On the other hand, it is often the case that test suite execution must finish within a fixed time budget. As test suite reduction is criterion-based, it is difficult to fit into a changing testing budget. Another difference between our work and the above studies is that we focus on the differences among test adequacy and compare the effectiveness among such criteria. This dimension is new, and has not been studied in related work.

Jiang et al. [15] studied the integration problem of test case prioritization and fault localization. Their results show that test case prioritization does have an impact on the effectiveness of fault localization techniques and that the random strategy is surprisingly effective. However, the work did not study to what extent test case prioritizations may generate test suites that existing fault localization techniques can use to locate faults effectively. Neither did the work investigate the impact of test adequacy criteria on statistical fault localization. Gonzalez-Sanchez et al. [11] proposed a new test case prioritization approach to maximize the improvement of the diagnostic information per test case. Their results showed that their technique could reduce the overall testing and debugging cost for some scenarios. They also did not examine the effect of adequate test suites on fault localization techniques.

There are plenty of studies on test case prioritization techniques. Srivastava and Thiagarajan [26] proposed a binary matching technique to compute the changes between program versions at the basic block level and prioritize test cases to cover greedily the affected program changes. Li et al. [21] conducted evaluations of various search-based algorithms for test cases prioritization. Their results show that several search-based algorithms are surprisingly effective. Leon et al. [20] also proposed failure-pursuit sampling techniques. They are based on the observation that failureinducing test cases tend to cluster together with respect to the code space of a program. Their failure-pursuit sampling uses one-per-cluster sampling to select the initial sample and, if a failure is found, its $k$ nearest neighbors will be selected and checked. If additional failures are found, the process will be repeated.

There are also studies on fault localization techniques closely related to the four techniques studied in our experiment. For example, Cleve and Zeller [6] proposed delta debugging, which automatically isolates failure-inducing inputs, generates cause-effect chains, and exposes the faults. Renieris and Reiss [24] observed that using the execution trace difference between a failed run and its nearest passed neighbor run is more effective than using other pairs for fault localization. Jeffrey et al. [12] proposed a value-profile based approach to ranking program statements according to their likelihood of being faulty. Zhang et al. [32] differentiated short-circuit evaluations of individual predicates in individual program statements and produced a set of evaluation sequences per predicate for fault localization. They found that the use of evaluation sequence can significantly improve existing fault localization techniques. Zhang et al. [31] used a network propagation approach, taking into consideration the error propagation phenomena along the edges of a program control flow graph. They rank the edges of the program control flow graph and propagate back the suspicious scores to the program statements (representing states).

Since our study is an integration of test case prioritization techniques and fault localization techniques, the experiment will grow steeply when we evaluate more fault localization techniques. We therefore focus on the four most typical fault localization techniques in our study so that the empirical study is manageable without losing representativeness. Similarly, in RQ3, we narrow down our study to compare between MC/DC- and branch-adequate test suites. Although we have restrained the scale of our study, to the best of our knowledge, it is the largest empirical study on this topic to date.

Researchers have studied the MC/DC adequacy criterion for a long time because of its significance. Yu et al. [30] compared MC/DC, MUMCUT, and other related coverage criteria for safety-critical software by formal and empirical analysis. Since MC/DC is the required coverage criterion for airborne software by FAA through the DO-178B standard, it is also extensively studied in the aeronautics industry. Dupuy et al. [8] conducted an empirical evaluation of the MC/DC adequacy criterion on the HETE-2 satellite software. They found that test cases generated using the MC/DCadequacy criterion detected important errors not detectable by functional testing. They further found that although MC/DC incurs more testing resources $(40 \%$ of the total testing time), the effort is worthwhile as it can detect errors that could not have been found by lower level structural coverage. The relationship between test case prioritization and the MC/DC adequacy criterion has also been studied by Jones and Harrold [18]. Our work does not analyze this dimension whereas the work of Jones and Harrold does not study the fault localization aspect.

\section{Concluding Remarks}

To select test cases from a huge input domain, testers use adequacy criteria to determine when to stop testing. Because the execution results and coverage information of the adequate test suite can be fed to fault localization techniques, the choice of adequacy criterion may have a significant impact on the effectiveness of fault localization.

We find from our study that stronger adequacy criteria may be more promising in supporting effective fault localization. In particular, we find that $\mathrm{MC} / \mathrm{DC}$ adequacy performs better than branch adequacy, which in turn performs better than statement adequacy. Furthermore, we conducted postmortem analysis on existing fault localization techniques and found that they still could not effectively 
narrow down the suspicious region of faults within one debug window of typical IDEs. The result shows that there are still large gaps in integrating various kinds of testing and debugging techniques so that they can be effectively used by developers uniformly. The result, however, indicates that MC/DC-adequate test suites can be more scalable and precise.

In terms of practice, there are a number of implications from the study. First, MC/DC is normally applied to safetycritical software. They have seldom been used in general. We have found from Tables 8 to 11 that as a stronger test adequacy is used, the probability of effective fault localization increases across all statistical fault localization techniques and across all subjects except applying Tarantula on the subject tot_info. In other words, the following conjecture holds, on average, for $97.7 \%$ of the 44 cases (four techniques with 11 subjects each):

Conjecture: Using a stronger test adequacy increases the probability of effective fault localization via the use of a prioritized adequate test suite.

Based on this validated conjecture, we recommend the use of MC/DC instead of branch adequacy, statement adequacy, or random selection as the criterion to construct adequate test suite if the goal of the testing-debugging integration is effective statistical fault localization.

We have further discussed in Section 3.5.1 that using MC/DC test suites can make the regression line in Fig. 1 to have a gentler slope and a smaller $y$-intercept than using branch- and statement-adequate test suites - that is, it is more scalable and has less fixed cost. Nonetheless, to the best of our knowledge, only weaker criteria are popularly supported by many industrial-strength tools. While this may indicate the favor and demand of the software industry, we have conjectured that such popular adequacy criteria could be too weak for fault localization. In our experiment, the adoption of a stronger adequacy criterion can lead to more effective integration of testing and debugging.

On the other hand, random testing can be effectively used, say, to crash a program. We believe that random testing and adequacy testing are useful for different purposes and are complementary to each other. Our paper focuses on the impact of adequate test suites on fault localization. It will be interesting to study other popular and useful testing techniques (such as data flow testing) and resolve their effective integration. It will also be interesting to study reliability testing and its integration with program debugging.

\section{References}

[1] Software considerations in airborne systems and equipment certification, DO-178B, RTCA, Washington, DC, 1992.

[2] The economic impacts of inadequate infrastructure for software testing, Final Report, National Institute of Standards and Technology, Gaithersburg, MD, 2002. Available from http://www.nist.gov/director/planning /upload/report02-3.pdf.
[3] R. Abreu, P. Zoeteweij, and A.J.C. van Gemund, On the accuracy of spectrum-based fault localization, in: Proceedings of the Testing: Academic and Industrial Conference: Practice And Research Techniques (TAICPART-MUTATION 2007), IEEE Computer Society, Los Alamitos, CA, 2007, pp. 89-98.

[4] B. Baudry, F. Fleurey, and Y. Le Traon, Improving test suites for efficient fault localization, in: Proceedings of the 28th International Conference on Software Engineering (ICSE 2006), ACM, New York, NY, 2006, pp. 82-91.

[5] C. Cadar, D. Dunbar, and D.R. Engler, KLEE: unassisted and automatic generation of high-coverage tests for complex systems programs, in: Proceedings of the 8th USENIX Conference on Operating Systems Design and Implementation (OSDI 2008), USENIX Association, Berkeley, CA, 2008, pp. 209-224.

[6] H. Cleve and A. Zeller, Locating causes of program failures, in: Proceedings of the 27th International Conference on Software Engineering (ICSE 2005), ACM, New York, NY, 2005, pp. 342-351.

[7] H. Do, S.G. Elbaum, and G. Rothermel, Supporting controlled experimentation with testing techniques: an infrastructure and its potential impact, Empirical Software Engineering 10 (4) (2005) 405-435.

[8] A. Dupuy and N. Leveson, An empirical evaluation of the MC/DC coverage criterion on the HETE-2 satellite software, in: Proceedings of the 19th Digital Avionics Systems Conference (DASC 2000), vol. 1, IEEE Computer Society, Los Alamitos, CA, 2000, pp. 1B6/11B6/7.

[9] S.G. Elbaum, A.G. Malishevsky, and G. Rothermel, Test case prioritization: a family of empirical studies, IEEE Transactions on Software Engineering 28 (2) (2002) 159-182.

[10] S.G. Elbaum, G. Rothermel, S. Kanduri, and A.G. Malishevsky, Selecting a cost-effective test case prioritization technique, Software Quality Control 12 (3) (2004) 185-210.

[11] A. Gonzalez-Sanchez, E. Piel, H.-G. Gross, and A.J.C. van Gemund, Prioritizing tests for software fault localization, in: Proceedings of the 10th International Conference on Quality Software (QSIC 2010), IEEE Computer Society, Los Alamitos, CA, 2010, pp. 42 51.

[12] D. Jeffrey, N. Gupta, and R. Gupta, Fault localization using value replacement, in: Proceedings of the 2008 ACM SIGSOFT International Symposium on Software Testing and Analysis (ISSTA 2008), ACM, New York, NY, 2008, pp. 167-178.

[13] B. Jiang and W.K. Chan, On the integration of test adequacy: test case prioritization and statistical fault 
localization, The 1st International Workshop on Program Debugging in China (IWPDC 2010), in Proceedings of the 10th International Conference on Quality Software (QSIC 2010), IEEE Computer Society, Los Alamitos, CA, 2010, 377-384.

[14] B. Jiang, W.K. Chan, and T.H. Tse, On practical adequate test suites for integrated test case prioritization and fault localization, in: Proceedings of the 11th International Conference on Quality Software (QSIC 2011), IEEE Computer Society, Los Alamitos, CA, 2011, pp. 21-30.

[15] B. Jiang, Z. Zhang, W.K. Chan, and T.H. Tse, Adaptive random test case prioritization, in: Proceedings of the 24th IEEE/ACM International Conference on Automated Software Engineering (ASE 2009), IEEE Computer Society, Los Alamitos, CA, 2009, pp. 233244.

[16] B. Jiang, Z. Zhang, W.K. Chan, T.H. Tse, and T.Y. Chen, How well does test case prioritization integrate with statistical fault localization?, Information and Software Technology 54 (7) (2012) 739-758.

[17] B. Jiang, Z. Zhang, T.H. Tse, and T.Y. Chen, How well do test case prioritization techniques support statistical fault localization, in: Proceedings of the 33rd Annual International Computer Software and Applications Conference (COMPSAC 2009), vol. 1, IEEE Computer Society, Los Alamitos, CA, 2009, pp. 99106.

[18] J.A. Jones and M.J. Harrold, Test-suite reduction and prioritization for modified condition/decision coverage, IEEE Transactions on Software Engineering 29 (3) (2003) 195-209.

[19] J.A. Jones, M.J. Harrold, and J. Stasko, Visualization of test information to assist fault localization, in: Proceedings of the 24th International Conference on Software Engineering (ICSE 2002), ACM, New York, NY, 2002, pp. 467-477.

[20] D. Leon, W. Masri, and A. Podgurski, An empirical evaluation of test case filtering techniques based on exercising complex information flows, in: Proceedings of the 27th International Conference on Software Engineering (ICSE 2005), ACM, New York, NY, 2005, pp. 412-421.

[21] Z. Li, M. Harman, and R.M. Hierons, Search algorithms for regression test case prioritization, IEEE Transactions on Software Engineering 33 (4) (2007) 225-237.

[22] B. Liblit, M. Naik, A.X. Zheng, A. Aiken, and M.I. Jordan, Scalable statistical bug isolation, in: Proceedings of the 2005 ACM SIGPLAN Conference on Programming Language Design and Implementation (PLDI 2005), ACM, New York, NY, 2005, pp. 15-26.
[23] C. Liu, X. Yan, L. Fei, J. Han, and S.P. Midkiff, SOBER: statistical model-based bug localization, in: Proceedings of the Joint 10th European Software Engineering Conference and 13th ACM SIGSOFT International Symposium on Foundations of Software Engineering (ESEC 2005/FSE-13), ACM, New York, NY, 2005, pp. 286-295.

[24] M. Renieris and S.P. Reiss, Fault localization with nearest neighbor queries, in: Proceedings of the 18th IEEE International Conference on Automated Software Engineering (ASE 2003), IEEE Computer Society, Los Alamitos, CA, 2003, pp. 30-39.

[25] G. Rothermel, R.H. Untch, C. Chu, and M.J. Harrold, Prioritizing test cases for regression testing, IEEE Transactions on Software Engineering 27 (10) (2001) 929-948.

[26] A. Srivastava and J. Thiagarajan, Effectively prioritizing tests in development environment, in: Proceedings of the 2002 ACM SIGSOFT International Symposium on Software Testing and Analysis (ISSTA 2002), ACM, New York, NY, 2002, pp. 97-106.

[27] W.E. Wong, V. Debroy, and B. Choi, A family of code coverage-based heuristics for effective fault localization, Journal of Systems and Software 83 (2) (2010) 188-208.

[28] W.E. Wong, J.R. Horgan, S. London, and H. Agrawal, A study of effective regression testing in practice, in: Proceedings of the 8th International Symposium on Software Reliability Engineering (ISSRE 1997), IEEE Computer Society, Los Alamitos, CA, 1997, pp. 264274.

[29] Y. Yu, J.A. Jones, and M.J. Harrold, An empirical study of the effects of test-suite reduction on fault localization, in: Proceedings of the 30th International Conference on Software Engineering (ICSE 2008), ACM, New York, NY, 2008, pp. 201-210.

[30] Y.T. Yu and M.F. Lau, A comparison of MC/DC, MUMCUT and several other coverage criteria for logical decisions, Journal of Systems and Software 79 (5) (2006) 577-590.

[31] Z. Zhang, W.K. Chan, T.H. Tse, B. Jiang, and X. Wang, Capturing propagation of infected program states, in: Proceedings of the 7th Joint Meeting of the European Software Engineering Conference and the ACM SIGSOFT International Symposium on Foundations of Software Engineering (ESEC 2009/FSE-17), ACM, New York, NY, 2009, pp. 43-52.

[32] Z. Zhang, B. Jiang, W.K. Chan, T.H. Tse, and X. Wang, Fault localization through evaluation sequences, Journal of Systems and Software 83 (2) (2010) 174-187. 